This item was submitted to Loughborough's Research Repository by the author.

Items in Figshare are protected by copyright, with all rights reserved, unless otherwise indicated.

\title{
The temperature dependence of the helical pitch in a cholesteric liquid crystal
}

PLEASE CITE THE PUBLISHED VERSION

https://doi.org/10.1080/00268976.2021.1881638

PUBLISHER

Taylor \& Francis

VERSION

AM (Accepted Manuscript)

PUBLISHER STATEMENT

This is an Accepted Manuscript of an article published by Taylor \& Francis in Molecular Physics on 10 Feb 2021, available online: http://www.tandfonline.com/10.1080/00268976.2021.1881638.

LICENCE

CC BY-NC-ND 4.0

\section{REPOSITORY RECORD}

Skutnik, Robert A, Jan-Christoph Eichler, Marco Mazza, and Martin Schoen. 2021. "The Temperature Dependence of the Helical Pitch in a Cholesteric Liquid Crystal”. Loughborough University.

https://hdl.handle.net/2134/13609100.v1. 


\title{
The temperature dependence of the helical pitch in a cholesteric liquid crystal
}

\author{
Robert A. Skutnik ${ }^{\mathrm{a}}$, Jan-Christoph Eichler ${ }^{\mathrm{a}}$, Marco G. Mazza ${ }^{\mathrm{b}, \mathrm{c}}$, and \\ Martin Schoen ${ }^{\mathrm{a}, \mathrm{d}}$ \\ ${ }^{a}$ Stranski-Laboratorium für Physikalische und Theoretische Chemie, Fakulät für Mathematik \\ und Naturwissenschaften, Technische Universität Berlin, Straße des 17. Juni 115, 10623 \\ Berlin, Germany; ${ }^{b}$ Interdisciplinary Centre for Mathematical Modelling and Department of \\ Mathematical Sciences, Loughborough University, Loughborough, Leicestershire LE11 3TU, \\ United Kingdom; 'Max Planck Institute for Dynamics and Self-Organization, Am Faßsberg \\ 17, 37077 Göttingen, Germany; ${ }^{\mathrm{d}}$ Department of Chemical Engineering, Imperial College \\ London, South Kensington Campus, London SW7 2AZ, United Kingdom
}

\section{ARTICLE HISTORY}

Compiled January 19, 2021

\begin{abstract}
We investigate the temperature dependence of the helical pitch of a cholesteric liquid crystal by means of Monte Carlo simulations. We carry out both lattice and off-lattice simulations to assess the impact of geometric and modelling constraints on the properties of the cholesteric phase. For the off-lattice simulations we develop boundary conditions commensurate with the cholesteric phase and derive an analytic expression for the helical wavenumber $q$ that works well qualitatively. We find that the common simplification of constraining the orientation of the mesogens to planes normal to the helical axis makes $q$ temperature-independent, as predicted by a mean-field theory of van der Meer et al. [J. Chem. Phys. 65, 3935 (1976)]. However, if mesogens are allowed to rotate in three dimensions, $q$ will increase with temperature, as the isotropic-cholesteric transition is approached from below, in agreement with experiments for a number of substances. Our simulations indicate that the temperature-independent $q$ is merely a consequence of the overly restricted orientational degrees of freedom to points on the unit circle in the model on which the mean-field theory is based.
\end{abstract}

\section{KEYWORDS}

Cholesteric liquid crystal, temperature dependence of helical pitch, lattice and off-lattice Monte Carlo simulation

\section{Introduction}

Chiral liquid crystals offer a unique arena where fluid behaviour, elasticity, order, topology, and optical activity all come to play an important role with fascinating results [15]. Historically, they were the first liquid-crystalline phase to be discovered, and since then they have inspired applications from optics [6] to nanostructures [3]. Because of their molecular shape and interactions chiral liquid crystals, also known as chiral nematics, develop a twist in their local nematic director. While on a very short scale they

Corresponding author: M. Schoen, e-mail: martin.schoen@tu-berlin.de 
appear nematically aligned, the director gradually rotates as one moves along a certain direction that emerges mesoscopically in the liquid, the helical axis. The direction in which the director rotates determines the handedness of the chiral fluid, and the length scale over which the director undergoes a full rotation determines the helical pitch. When the pitch is comparable to the wavelength of visible light, the optical activity of a chiral liquid crystal becomes visible, which has engendered applications as lasers [7] and photonic band-gap devices [8].

Despite the technological progress that occurred in the past decades, there is still considerable uncertainty about the theoretical foundations for the temperature dependence of the cholesteric pitch, even in the simplest conditions of a bulk fluid. In most compounds derived from cholesterol the pitch increases with decreasing temperature [9], which is due to the onset of smectic layering at lower temperatures [10]; smectic order is not compatible with the twist deformation, and thus the pitch increases upon lowering temperature. Simulations can help unravel the role of different physical effects. Varga and Jackson [11] found a pitch increasing with temperature due to a kinetic 'unwinding' of the cholesteric helix upon approaching the isotropiccholesteric phase transition, which can be observed experimentally in suspensions of $f d$ viruses [12]. In lyotropic systems the pitch can also have a nonmonotonic temperature dependence due to entropic unwinding of the cholesteric helix [13].

Theoretical models have also found contrasting results depending on the type of approximations used which are often of a very drastic nature. In a landmark paper in 1976, van der Meer et al. [14] derived a mean-field theory and showed that $q$ is independent of temperature in the simple version of their theory. In their treatment the orientations of the mesogens are restricted to the $\mathcal{S}^{1}$ sphere, that is, the tips of their orientation vectors can only move along the unit circle, but they may still change continuously.

Prompted by the observation that experimentally $q$ is generally not independent of temperature $T$, van der Meer et al. [14] proposed a more sophisticated version of their mean-field theory in which such a temperature dependence would arise through various order parameters. Unfortunately, the performance of this theory had not been tested in the original paper by van der Meer et al. [14] due to lacking data at the time.

About twenty years later the mean-field theory by van der Meer et al. [14] had been tested in Monte Carlo (MC) simulations by Luckhurst et al. [15] and by Memmer and Janssen $[16,17]$. In both studies $[15,16]$ the $\mathcal{S}^{1}$-model was employed and the helical wavenumber turned out to be temperature-independent in line with the simple version of the mean-field theory [14]. In the present work we confirm these earlier findings. However, employing the $\mathcal{S}^{2}$-model we demonstrate here that the absence of a temperature dependence of $q$ is merely a consequence of the rather severe and unphysical restriction of the orientations to the unit circle in the $\mathcal{S}^{1}$-model. In the $\mathcal{S}^{2}$-model the tips of the unit vectors describing the orientations of the mesogens correspond to points on the surface of the unit sphere instead.

The rest of the article is organised as follows. In Section 2 we introduce the model that we solve using both theory and MC simulations. Section 3 described the observables used to characterise the cholesteric state. Section 4 shows the results for both lattice and off-lattice MC simulations. Finally, in Section 5 we discuss our results and gather our conclusions. 


\section{Model}

We consider a chiral liquid crystal composed of $N$ molecules (mesogens). These mesogens are located either at the sites of a simple-cubic lattice or move along continuous trajectories in space. The lattice consists of $n$ regularly spaced planes stacked along the $z$-axis of a fixed laboratory reference system; each plane comprises $n \times n$ discrete sites in the $x-y$ plane and therefore $N=n^{3}$.

\subsection{The interaction potential for the general model}

The intermolecular interactions between a pair of mesogens can be described by an orientation-dependent potential $u$ that we decompose into an isotropic contribution $u_{\text {iso }}$ and into an anisotropic one $u_{\text {aniso }}$ according to

$$
u\left(\boldsymbol{r}_{12}, \Omega_{1}, \Omega_{2}\right)=u_{\text {iso }}\left(r_{12}\right)+u_{\text {aniso }}\left(\boldsymbol{r}_{12}, \Omega_{1}, \Omega_{2}\right) .
$$

In Equation (1), $\boldsymbol{r}_{12}=\boldsymbol{r}_{1}-\boldsymbol{r}_{2}$ is the distance vector connecting the centres of mass of mesogens 1 and 2 located at $\boldsymbol{r}_{1}$ and $\boldsymbol{r}_{2}$, respectively; $r_{12}=\left|\boldsymbol{r}_{12}\right|$ and $\Omega_{1}$ and $\Omega_{2}$ are sets of three Euler angles $\left(\phi^{\prime}, \theta^{\prime}, \chi^{\prime}\right)$ where we employ the convention introduced in Section A.2 of the book by Gray and Gubbins [18]. The prime indicates that we are referring to either mesogen 1 or 2 .

We take $u_{\text {iso }}$ to be represented by the soft-sphere potential

$$
u_{\text {iso }}\left(r_{12}\right)=4 \varepsilon\left(\frac{\sigma}{r_{12}}\right)^{12} \text {. }
$$

The parameters $\varepsilon$ and $\sigma$ define the energy and length scales pertinent to the model, respectively (see also Appendix A).

The anisotropic part of the interaction potential $u$ in Equation (1) can be expanded in part (see discussion in Section 2.1.2) according to

$$
u_{\text {aniso }}\left(\boldsymbol{r}_{12}, \Omega_{1}, \Omega_{2}\right)=\sum_{l_{1} l_{2} l} \sum_{n_{1} n_{2}} u_{l_{1} l_{2} l}^{n_{1} n_{2}}\left(r_{12}\right) \mathcal{S}_{l_{1} l_{2} l}^{n_{1} n_{2}}\left(\Omega_{1}, \Omega_{2}, \Omega\right)
$$

where $\left\{u_{l_{1} l_{2} l}^{n_{1} n_{2}}\right\}$ is a set of expansion coefficients that depend only on $r_{12}$ and the real, rotationally invariant functions $\left\{\mathcal{S}_{l_{1} l_{2} l}^{n_{1} n_{2}}\right\}$ form the basis in which the expansion is carried out. Mathematical details of the expansion are deferred to Appendix A. In Equation (3), $\Omega$ specifies the orientation of $\widehat{\boldsymbol{r}}_{12}=\boldsymbol{r}_{12} / r_{12}$ in the laboratory reference frame. Here and throughout the entire manuscript the caret is used to indicate unit vectors.

Focusing henceforth on dispersion interactions, Gray and Gubbins [18] show in Chapter 2.6 of their book that the expansion coefficients $\left\{u_{l_{1} l_{2} l}^{n_{1} n_{2}}\right\}$ in Equation (3) have units of energy $\times$ length $^{-6}$. Different expansion coefficients involve different combinations of material-specific parameters and other trivial numerical factors (see Equation (2.276) of the book by Gray and Gubbins [18]). However, the distance dependence of the expansion coefficients can be assumed to be the same regardless of the values of $l_{1}, l_{2}$, and $l$ such that they are all proportional to $-\varepsilon\left(\sigma / r_{12}\right)^{6}$. All other combinations of molecular properties and trivial numerical prefactors arising in the expression for the expansion coefficients $\left\{u_{l_{1} l_{2} l}^{n_{1} n_{2}}\right\}$ are lumped together in various dimensionless coupling constants to be specified below (see discussion in Chapter 2.6 of the book by Gray and 
Gubbins [18]).

However, because our mesogens can be perceived as spheroids, only the subspace $\omega^{\prime}=\left(\phi^{\prime}, \theta^{\prime}\right)$ is sufficient to describe their orientations in a laboratory reference frame. As we discuss in greater detail in the subsequent Sections 2.1.1 and 2.1.2 the expansion in Equation (3) then eventually permits us to recast $u_{\text {aniso }}$ as

$$
u_{\text {aniso }}\left(\boldsymbol{r}_{12}, \omega_{1}, \omega_{2}\right)=-4 \varepsilon\left(\frac{\sigma}{r_{12}}\right)^{6} \Psi\left(\omega_{1}, \omega_{2}, \omega\right),
$$

where the "anisotropy function" $\Psi$ accounts for the orientation dependence of the interactions. In Equation (4), $\omega=(\phi, \theta)$ specifies the orientation of $\widehat{\boldsymbol{r}}_{12}=\boldsymbol{r}_{12} / r_{12}$ in a space-fixed reference frame. We now turn to a discussion of the specific form of the anisotropy function in our general model which can be split formally into an isotropic, achiral, and chiral contribution according to

$$
\Psi\left(\omega_{1}, \omega_{2}, \omega\right)=\varepsilon_{0}^{\prime}+\Psi_{\mathrm{a}}\left(\omega_{1}, \omega_{2}\right)+\Psi_{\mathrm{c}}\left(\omega_{1}, \omega_{2}, \omega\right),
$$

where the constant term on the right-hand side is due to $\mathcal{S}_{000}=1$ [19].

\subsubsection{Orientation dependence of the achiral anisotropic interactions}

We begin with the contribution of the achiral degrees of freedom to the orientation dependence of the anisotropic interaction between a pair of mesogens. As we discuss in more detail in Appendix A we can cast $\Psi_{\mathrm{a}}$ more explicitly as

$$
\Psi_{\mathrm{a}}\left(\omega_{1}, \omega_{2}\right)=\varepsilon_{2}^{\prime} \mathcal{S}_{220}=\varepsilon_{2}^{\prime} P_{2}(x),
$$

where $x \equiv \cos \gamma=\widehat{\boldsymbol{u}}_{1} \cdot \widehat{\boldsymbol{u}}_{2}, \widehat{\boldsymbol{u}}_{i}(i=1,2)$ is a unit vector describing the orientation of mesogen $i, P_{2}=\frac{1}{2}\left(3 x^{2}-1\right)$ is the second Legendre polynomial, and $\varepsilon_{2}^{\prime}$ is a dimensionless coupling constant. To arrive at the far right-hand side of Equation (6), Equation (A5) has also been invoked. We dropped the arguments of the rotational invariants as well as their upper indices $n_{1}$ and $n_{2}$ because both integers are zero throughout this work as we are strictly limiting ourselves to spheroidal mesogens. We note in passing that the function $\Psi_{\mathrm{a}}$ in Equation (6) is the same as that chosen by Maier and Saupe in their celebrated, classic model of a nematic liquid crystal [20].

However, for reasons to be explained shortly we prefer to replace Equation (6) with the equivalent expression

$$
\Psi_{\mathrm{a}}\left(\omega_{1}, \omega_{2}\right)=\varepsilon_{2}\left(\widehat{\boldsymbol{u}}_{1} \cdot \widehat{\boldsymbol{u}}_{2}\right)^{2},
$$

where the coupling constant $\varepsilon_{2}$ has to be large enough to induce sufficiently strong local uniaxial order. In spherical coordinates, the unit vectors $\widehat{\boldsymbol{u}}_{1}$ and $\widehat{\boldsymbol{u}}_{2}$ are specified by

$$
\widehat{\boldsymbol{u}}_{i} \equiv \widehat{\boldsymbol{u}}\left(\omega_{i}\right)=\left(\begin{array}{c}
u_{\mathrm{x} i} \\
u_{\mathrm{y} i} \\
u_{\mathrm{z} i}
\end{array}\right)=\left(\begin{array}{c}
\sin \theta_{i} \cos \phi_{i} \\
\sin \theta_{i} \sin \phi_{i} \\
\cos \theta_{i}
\end{array}\right), \quad i=1,2
$$

where $\phi^{\prime}$ and $\theta^{\prime}$ denote the azimuthal and the polar angle, respectively. We introduced new dimensionless coupling constants $\varepsilon_{0}^{\prime}=\varepsilon_{0}=1$ in Equation (5) and $\varepsilon_{2} \geq 0$. The 
coupling constants $\varepsilon_{2}^{\prime}$ and $\varepsilon_{2}$ are then related through a simple expression. The choice $\varepsilon_{2}>0$ stabilises the nematic phase.

The reason for preferring Equation (7) over its equivalent counterpart in Equation (6) is that we wish to compare our lattice MC simulations with predictions of a mean-field theory devised by van der Meer et al. [14] quite some time ago. This theory is based upon an achiral contribution to $u$ given by Equation (7).

\subsubsection{Orientation dependence of the chiral anisotropic interactions}

Whereas a model potential solely based upon Equation (7) promotes the formation of a globally nematic phase, it is insufficient for the formation of chiral phases. This is because $\Psi_{\mathrm{a}}$ is invariant with respect to the transformation $\boldsymbol{r}_{12} \rightarrow-\boldsymbol{r}_{12}$. To abandon this inversion symmetry requires additional terms to appear. These terms ought to be pseudo-scalar in nature [14] because $u$ is a scalar quantity. Moreover, the nematic character of ordered phases imposed by $\Psi_{\mathrm{n}}$ should be preserved at the same time but only locally.

Unfortunately, a unified treatment of chiral interactions within the framework just delineated in Section 2.1.1 turns out to be impossible. This is because for dispersion interactions the expansion coefficients introduced in Equation (3) are proportional to the Clebsch-Gordan coefficient $C(22 l ; 000)[18]$. We demonstrate in Appendix A that the simplest pseudo-scalar needed requires $l=1$. However, because of the parity relation for the Clebsch-Gordan coefficients $l_{1}+l_{2}+l$ must be an even integer. In other words, chiral dispersion interactions do not exist. This subtlety has been missed in all previous studies [11, 14-17, 21-23] of chiral liquid crystals within the framework of Maier-Saupe-like models.

Interestingly, it was already pointed out by Stone [19] that rotational invariants of "... odd total rank ..." $l_{1}+l_{2}+l$ "... do not occur in the description of quantities such as energy which are invariant under inversion. However, they do occur in the theory of optical activity ..." [24, 25]. Stone [25] then demonstrates that the rotational invariant $\mathcal{S}_{221}$ is central to the theory of differential light scattering.

In fact, as Barron and Buckingham [26] pointed out "... the absolute configuration..." (shown in their Figure 3) "... leads to positive Rayleigh CIDâA $\breve{Z}{ }^{1}{ }^{\text {and }}$ a positive optical rotation (a clockwise rotation when viewed toward the source of the light) at the same transparent wavelength." The cartoon of a typical molecular conformation shown by these latter authors in Figure 3 of their paper is, in fact, akin to that typical of a cholesteric phase (see Figure 2 of Ref. [14]). We derive an explicit expression for $\mathcal{S}_{221}$ in Appendix A.

From the perspective of computer simulations and because of the appearance of the rotational invariant $\mathcal{S}_{221}$ it is convenient to introduce a new function [see Equations (A6)-(A9)]

$$
\begin{aligned}
\Psi_{\mathrm{c}}\left(\omega_{1}, \omega_{2}, \omega\right) & =\varepsilon_{1}^{\prime} \mathcal{S}_{221} \\
& =\varepsilon_{1}\left(\widehat{\boldsymbol{u}}_{1} \cdot \widehat{\boldsymbol{u}}_{2}\right)\left(\widehat{\boldsymbol{u}}_{1} \times \widehat{\boldsymbol{u}}_{2}\right) \cdot \widehat{\boldsymbol{r}}_{12}
\end{aligned}
$$

defined in parallel with $\Psi_{\mathrm{a}}$ in Equation (7). In Equation (9), $\varepsilon_{1}$ is yet another dimensionless coupling constant. As in Section 2.1.1, the two coupling constants in Equation (9) are again trivially related.

It has been demonstrated repeatedly $[11,14-16,21-23,27]$ that a term proportional

\footnotetext{
${ }^{1}$ CID: circular intensity differential
} 
to $\mathcal{S}_{221}$ is sufficient to promote the formation of a cholesteric phase. However, on the basis of their mean-field theory van der Meer et al. [14] have argued that a higher-order achiral contribution proportional to $\left(\widehat{\boldsymbol{u}}_{1} \cdot \widehat{\boldsymbol{u}}_{2}\right)^{4}$ as well as a higher-order pseudo scalar quantity proportional to $\left(\widehat{\boldsymbol{u}}_{1} \cdot \widehat{\boldsymbol{u}}_{2}\right)^{3}\left(\widehat{\boldsymbol{u}}_{1} \times \widehat{\boldsymbol{u}}_{2}\right) \cdot \widehat{\boldsymbol{r}}_{12}$ are required to describe a temperature dependence of the pitch of the cholesteric helix. As we will be demonstrating below this is not the case. Using Equations (7) and (9) we can reexpress the anisotropy function in Equation (5) as

$$
\Psi\left(\omega_{1}, \omega_{2}, \omega\right)=1+\varepsilon_{2}\left(\widehat{\boldsymbol{u}}_{1} \cdot \widehat{\boldsymbol{u}}_{2}\right)^{2}+\varepsilon_{1}\left(\widehat{\boldsymbol{u}}_{1} \cdot \widehat{\boldsymbol{u}}_{2}\right)\left(\widehat{\boldsymbol{u}}_{1} \times \widehat{\boldsymbol{u}}_{2}\right) \cdot \widehat{\boldsymbol{r}}_{12}
$$

for the general model employed in this work.

\subsection{Specialised versions of the general model}

We are now turning to a discussion of specialised versions of the general model introduced in Sections 2.1.1 and 2.1.2. These are either discretised on a lattice of a simple-cubic symmetry or permit mesogens to move along continuous trajectories.

We begin by introducing the off-lattice model using Equations (1), (2), (4), and (10)

$$
\begin{aligned}
u\left(\boldsymbol{r}_{12}, \omega_{1}, \omega_{2}\right)= & \underbrace{4 \varepsilon\left[\left(\frac{\sigma}{r_{12}}\right)^{12}-\left(\frac{\sigma}{r_{12}}\right)^{6}\right]}_{u_{\mathrm{LJ}}\left(r_{12}\right)} \\
& -4 \varepsilon\left(\frac{\sigma}{r_{12}}\right)^{6}\left\{\Psi_{\mathrm{a}}\left(\omega_{1}, \omega_{2}\right)+\Psi_{\mathrm{c}}\left(\omega_{1}, \omega_{2}, \omega\right)\right\} .
\end{aligned}
$$

We see that $\sigma$ can be interpreted as a measure of the size of the spherically symmetric core of the mesogens; the minimum of $u_{\mathrm{LJ}}$ is located at $r_{\min }=2^{1 / 6} \sigma$ and therefore $\varepsilon$ is the depth of the attractive well of the Lennard-Jones potential $u_{\mathrm{LJ}}$ introduced in Equation (11).

However, it is known [22] that a model based upon the interaction potential introduced in Equation (11) allows for a formation of a stable cholesteric phase only for very small values of $\varepsilon_{1}$ and if additional precautions such as confining walls are introduced; for moderate to high values of $\varepsilon_{1}$ blue phases turn out to surpass the cholesteric one in terms of stability [22]. To cure this unwanted feature of our model and to abolish the use of confining walls we replace $\Psi_{\mathrm{c}}$ in Equation (11) by

$$
\Psi_{\mathrm{c}}^{\prime}\left(\omega_{1}, \omega_{2}, \omega\right)=\varepsilon_{1}\left(\widehat{\boldsymbol{r}}_{12} \cdot \widehat{\boldsymbol{e}}_{\mathrm{z}}\right)^{2}\left(\widehat{\boldsymbol{u}}_{1} \cdot \widehat{\boldsymbol{u}}_{2}\right)\left(\widehat{\boldsymbol{u}}_{1} \times \widehat{\boldsymbol{u}}_{2}\right) \cdot \widehat{\boldsymbol{r}}_{12} .
$$

The additional prefactor $\left(\widehat{\boldsymbol{r}}_{12} \cdot \widehat{\boldsymbol{e}}_{\mathrm{z}}\right)^{2}$ tends to stabilise a cholesteric helix propagating in the $\pm z$-direction. It is important to realise that this additional factor does not completely restrict the orientations of the mesogens to the $x-y$ plane but only weights these orientations energetically slightly more favourably.

However, we wish to point out that the prefactor $\left(\widehat{\boldsymbol{r}}_{12} \cdot \widehat{\boldsymbol{e}}_{\mathrm{z}}\right)^{2}$ in Equation (12) is introduced "by hand", so to speak, to serve two main purposes. First, it suppresses the unwanted blue phases in favour of the cholesteric phase which is energetically stabilised. Second, it aligns the cholesteric helix with the $z$-axis of the laboratory reference frame in a controllable and reproducible fashion. In the longer run, it may be possible to stabilise the cholesteric phase without this prefactor by using a model with biaxial 
symmetry as some preliminary simulations indicate. However, further discussions of this aspect are deferred to a separate publication.

Clearly, the prefactor in Equation (12) plays the role of a weak external field and as such should normally not appear in an intermolecular potential. Alternatively, one could confine the liquid crystal between planar solid substrates with or without additional anchoring such that the molecules tend to align with a specific direction at one substrate but can freely rotate at the other one [22].

This approach has the disadvantage that due to the interaction with the substrate the liquid crystal becomes inhomogeneous over distances from the substrate that are relatively large even for short-range potentials. The inhomogeneity is caused by packing effects (i.e., layering) that tend to propagate into the liquid crystal. Therefore, if one intends to study a cholesteric bulk material (as we do in this work) one can average any system properties only over that portion of the liquid crystal that is sufficiently far removed from both substrates where the system is homogeneous. In a computer simulation one would have to employ much larger systems that are consequently more costly computationally. With our present approach inhomogeneity is not an issue.

Another possibility that avoids both the additional prefactor in Equation (12) or the use of confining solid substrates would be to prepare the system such that the pitch of the cholesteric helix matches the dimensions of the computational cell so that the helix is not exposed to spurious stress on account of a possible mismatch of the two length scales. The obvious disadvantage here is that one needs to know beforehand what the pitch will be under the given thermodynamic conditions to choose the right dimensions of the computational cell. Neither one of these approaches seems to have advantages over using the modified potential we introduce in Equation (12).

Turning now to the lattice versions of our model we restrict the interaction between mesogens to nearest-neighbour sites on the lattice. On account of the simple-cubic symmetry of the lattice each mesogen has six nearest neighbours: four located in the same lattice $(x-y)$ plane and two directly above and below in the two adjacent lattice planes. Taking the nearest-neighbour distance $r_{12}$ on the lattice equal to $\sigma$ we obtain from Equation (11) the expression

$$
u\left(\omega_{1}, \omega_{2}, \omega\right)=-4 \varepsilon\left[\Psi_{\mathrm{a}}\left(\omega_{1}, \omega_{2}\right)+\Psi_{\mathrm{c}}^{\prime}\left(\omega_{1}, \omega_{2}, \omega\right)\right] .
$$

At the same time, the chiral contribution to $u$ acts only between nearest-neighbour mesogens located at sites in adjacent lattice planes at different positions along the $\pm z$-axis. This is because on the lattice and with the restriction to nearest-neighbour interactions $\left(\widehat{\boldsymbol{r}}_{12} \cdot \widehat{\boldsymbol{e}}_{\mathrm{z}}\right)^{2}$ is either zero $\left(\widehat{\boldsymbol{r}}_{12} \perp \widehat{\boldsymbol{e}}_{\mathrm{z}}\right)$ or one $\left(\widehat{\boldsymbol{r}}_{12} \| \widehat{\boldsymbol{e}}_{\mathrm{z}}\right)$. In this sense our versions of the lattice models is completely equivalent to the ones employed by Luckhurst et al. [15] and was utilised by Memmer and Janssen [16].

However, it needs to be borne in mind that the unit vectors $\widehat{\boldsymbol{u}}_{i}$ that specify the orientations of the mesogens on the lattice are defined as points $\widehat{\boldsymbol{x}}$ on the surface of the unit 2-sphere $\mathcal{S}^{2} \equiv\left\{\widehat{\boldsymbol{x}} \in \mathbb{R}^{3} \mid\|\widehat{\boldsymbol{x}}\|=1\right\}$ as an inspection of Equation (8) clearly shows. Thus, even though chiral interactions are completely restricted to mesogens located in adjacent lattice planes, the orientations of the mesogens can to a certain extent escape to the third dimension as it were and are not solely and exclusively forced to lie in the $x-y$ plane. We therefore refer to this version of the lattice model as the " $\mathcal{S}^{2}$-model" henceforth.

In their lattice MC simulations Luckhurst et al. [15] and Memmer and Janssen [16] use an even more restrictive model system. In these simulations the orientations of the molecules are defined as points on the surface of the $\mathcal{S}^{1}$ sphere (i.e. on the 
equator of the $\mathcal{S}^{2}$ sphere). The orientation vectors $\widehat{\boldsymbol{u}}_{i}$ are given by Equation (8) for the special case $\theta=\frac{\pi}{2}$. We shall be demonstrating below that this severe restriction has consequences for the temperature dependence of the helical wavenumber and also causes other unphysical properties. We shall be referring to the model studied by Luckhurst et al. [15] and by Memmer and Janssen [16] as the $\mathcal{S}^{1}$-model henceforth. The $\mathcal{S}^{1}$-model has also been employed in the mean-field theory of van der Meer et al. [14] which is not restricted to lattices though.

\section{Properties of cholesteric phases}

\subsection{Order parameters}

To analyse structural properties and to quantify the degree of order in the cholesteric phase we now introduce suitable order parameters and other measures of chiral order. On account of the locally nematic structure we introduce the local nematic order parameter

$$
\begin{aligned}
S\left(z_{k}\right) & =\left\langle\frac{1}{N_{k}} \sum_{i=1}^{N_{k}} \frac{1}{2}\left[3 \cos ^{2} \theta_{i}\left(z_{k}\right)-1\right]\right\rangle \\
& =\left\langle\frac{1}{N_{k}} \sum_{i=1}^{N_{k}} \frac{1}{2}\left[3\left(\widehat{\boldsymbol{n}}\left(z_{k}\right) \cdot \widehat{\boldsymbol{u}}_{i}\right)^{2}-1\right]\right\rangle, \quad k=1, \ldots, n,
\end{aligned}
$$

where $\langle\ldots\rangle$ indicates an ensemble average. For both the $\mathcal{S}^{1}$ - and the $\mathcal{S}^{2}$-models $z_{k}$ is the position of lattice plane $k$ along the $z$-axis. On the lattice, the individual planes are separated by a distance $\delta_{\mathrm{z}}=\sigma$; for the off-lattice model $z_{k}$ denotes the position of the centre of a thin slice of variable width $\delta_{z}$. Likewise, $N_{k}$ is either the number of mesogens located in that lattice plane or within the slice; there is a total of $n$ such planes or slices. In this expression, $\widehat{\boldsymbol{n}}$ is the local nematic director, and $\widehat{\boldsymbol{u}}_{i}$ has been introduced in Equation (8).

Unfortunately, as has been stressed by Eppenga and Frenkel [28] quite some time ago, Equation (14) is useless from a computational point of view because $\widehat{\boldsymbol{n}}$ is unknown a priori. However, using Equation (B.15) of Ref. [18] it is easy to realise that $\boldsymbol{b} \cdot(\boldsymbol{a a}) \cdot \boldsymbol{b}=$ $(\boldsymbol{a} \cdot \boldsymbol{b})^{2}$ where $\boldsymbol{a}$ and $\boldsymbol{b}$ are vectors and $\boldsymbol{a} \boldsymbol{a}$ denotes a second-rank tensor (i.e., a dyad). This allows us to rewrite Equation (14) as [28]

$$
S\left(z_{k}\right)=\left\langle\frac{1}{N_{k}} \sum_{i=1}^{N_{k}} \widehat{\boldsymbol{n}}\left(z_{k}\right) \cdot \mathbf{Q}_{i} \cdot \widehat{\boldsymbol{n}}\left(z_{k}\right)\right\rangle,
$$

where the Hermitian and traceless tensor $\mathbf{Q}_{i}$ is defined as

$$
\mathbf{Q}_{i}=\frac{1}{2}\left[3 \widehat{\boldsymbol{u}}_{i} \widehat{\boldsymbol{u}}_{i}-\mathbf{1}\right] .
$$

To proceed it is convenient to introduce

$$
\mathbf{Q}\left(z_{k}\right)=\frac{1}{N_{k}} \sum_{i=1}^{N_{k}} \mathbf{Q}_{i}, \quad k=1, \ldots, n
$$


which satisfies the eigenvalue equation

$$
\mathbf{Q}\left(z_{k}\right) \widehat{\boldsymbol{n}}_{ \pm 0}\left(z_{k}\right)=\lambda_{ \pm 0}\left(z_{k}\right) \widehat{\boldsymbol{n}}_{ \pm 0}\left(z_{k}\right),
$$

where $\lambda_{-}, \lambda_{0}$, and $\lambda_{+}$are the three eigenvalues of $\mathbf{Q}$ associated with the respective eigenvectors $\widehat{\boldsymbol{n}}_{-}, \widehat{\boldsymbol{n}}_{0}$, and $\widehat{\boldsymbol{n}}_{+}$. In general, these eigenvalues can be sorted according to the inequality $\lambda_{-}<\lambda_{0}<\lambda_{+}$.

In an ideal cholesteric phase and on account of its local uniaxial symmetry one expects $\lambda_{-}=\lambda_{0}<\lambda_{+}$. We follow common practice [28] and define the local nematic order parameter via

$$
S\left(z_{k}\right)=\left\langle\lambda_{+}\left(z_{k}\right)\right\rangle
$$

and take the set of associated local eigenvectors $\left\{\widehat{\boldsymbol{n}}_{+}\right\}$as local nematic directors $\{\widehat{\boldsymbol{n}}\}$. This expression is readily obtained by using Equations (15)-(18) and by also utilising the fact that $\widehat{\boldsymbol{n}}$ is a unit vector. Because in the cholesteric phase we quantify the local nematic order with respect to the set of local nematic directors $\{\widehat{\boldsymbol{n}}\}$, we obtain the global nematic order parameter from the expression

$$
S=\frac{1}{n} \sum_{k=1}^{n} S\left(z_{k}\right) .
$$

At this stage it seems worthwhile to stress that the local nematic director is obtained for each given configuration by solving Equation (18). However, in practice the above equality between $\lambda_{-}$and $\lambda_{0}$ will not hold exactly. It will thus be useful to introduce the biaxiality order parameter

$$
\xi \equiv \frac{1}{n} \sum_{k=1}^{n}\left[\left\langle\lambda_{0}\left(z_{k}\right)\right\rangle-\left\langle\lambda_{-}\left(z_{k}\right)\right\rangle\right]
$$

to quantify the deviation from the local uniaxial symmetry of the cholesteric phase [29]. We will show in Section 4 that for the $\mathcal{S}^{2}$-model and for the off-lattice model on the one hand and for the system sizes chosen in this work, $\xi=\mathcal{O}\left(10^{-2}-10^{-1}\right)$ due to the inevitable finiteness of the systems under study. In the $\mathcal{S}^{1}$-model, on the other hand, $\xi=\mathcal{O}(1)$.

At this point it is useful to introduce yet another order parameter which measures the degree of alignment of the mesogens with the $\widehat{\boldsymbol{n}}_{-}-\widehat{\boldsymbol{n}}_{0}$ plane. This order parameter is defined as [30]

$$
S_{\theta}=-\frac{1}{n} \sum_{k=1}^{n}\left\langle\frac{1}{N_{k}} \sum_{i=1}^{N_{k}} \cos \left(2 \theta_{i}\right)\right\rangle .
$$

One can easily show that in the isotropic phase, $S_{\theta}=\frac{1}{3}$ [30] whereas $S_{\theta}=1$ in a perfectly ordered cholesteric (locally nematic) phase in which $\theta_{i}=\frac{\pi}{2}$ irrespective of $i$. The reader should appreciate the difference between $S_{\theta}$ in Equation (22) and the two-dimensional nematic order parameter introduced by Frenkel and Eppenga [31]. The latter is based upon the alignment tensor for a two-dimensional system (see Equation (9) of Ref. [31]) which differs from Equation (16). 


\subsection{Structure}

Another quantity of interest is the orientation distribution function (odf). As explained in detail in Refs. [30, 32] it is advantageous to rotate the orientations of the mesogens in the $\mathcal{S}^{2}$ - and in the off-lattice model such that they lie in the $x-y$ plane with their tips located on the equator of the unit sphere $\mathcal{S}^{2}$. Because the eigensystem of $\mathbf{Q}$ consists of three vectors that are initially not necessarily orthogonal to the equatorial plane of the $\mathcal{S}^{2}$ sphere, a rotation of the orientations of the mesogens is needed. The rotation is effected by a rotation matrix $\mathbf{R}$ which can be set up as a direction cosine matrix between vectors of the standard basis $\left\{\widehat{\boldsymbol{e}}_{x}, \widehat{\boldsymbol{e}}_{y}, \widehat{\boldsymbol{e}}_{z}\right\}$ and components of the three eigenvectors $\left\{\widehat{\boldsymbol{n}}_{-}, \widehat{\boldsymbol{n}}_{0}, \widehat{\boldsymbol{n}}_{+}\right\}$.

We can then define the odf in a lattice plane or in the slice at $z_{k}$ as

$$
\alpha_{k}(\omega)=\frac{\sum_{i=1}^{N_{k}}\left\langle\delta\left(\omega-\widetilde{\omega}_{i}\right)-\delta\left(\omega-\widetilde{\widetilde{\omega}}_{i}\right)\right\rangle}{\int_{0}^{2 \pi} \int_{0}^{\pi} \sin \theta \mathrm{d} \theta \mathrm{d} \phi \sum_{i=1}^{N_{k}}\left[\delta\left(\omega-\widetilde{\omega}_{i}\right)-\delta\left(\omega-\widetilde{\widetilde{\omega}}_{i}\right)\right]} .
$$

Because for each $k, \alpha_{k}$ is calculated using a rotating reference frame we define $\alpha=n^{-1} \sum_{k=1}^{n} \alpha_{k}$ to enhance the statistical accuracy. In Equation (23), $\widetilde{\widetilde{\omega}} i=$ $\left(\pi-\widetilde{\theta}_{i}, \pi+\widetilde{\phi}_{i}\right)$ because of the spheroidal symmetry of the mesogens. The odf is normalised such that $\int \mathrm{d} \omega \alpha_{k}=1$. Because of this normalisation $\alpha_{k}=\frac{1}{4 \pi}$ in the isotropic phase $(k=1, \ldots, n)$ of the $\mathcal{S}^{2}$ - and of the off-lattice model; the corresponding value for the $\mathcal{S}^{1}$-model is $\alpha_{k}=\frac{1}{2 \pi}$ instead.

A quantity that is central to this work is the helical wavenumber $q$. It is related to the local nematic director $\widehat{\boldsymbol{n}}$ which rotates around the propagation direction of the cholesteric helix. Expressing $\widehat{\boldsymbol{n}}$ in spherical coordinates we can describe the local nematic director in the cholesteric phase by [29]

$$
\widehat{\boldsymbol{n}}\left(z_{k}\right)=\left(\begin{array}{c}
\sin \theta\left(z_{k}\right) \cos \phi\left(z_{k}\right) \\
\sin \theta\left(z_{k}\right) \sin \phi\left(z_{k}\right) \\
\cos \theta\left(z_{k}\right)
\end{array}\right) \stackrel{\theta=\pi / 2}{=}\left(\begin{array}{c}
\cos \phi\left(z_{k}\right) \\
\sin \phi\left(z_{k}\right) \\
0
\end{array}\right)=\left(\begin{array}{c}
\cos \left(2 \pi z_{k} / p\right) \\
\sin \left(2 \pi z_{k} / p\right) \\
0
\end{array}\right)
$$

where $p=\frac{2 \pi}{q}$ is the pitch of the helix. In addition, we assume $\theta=\frac{\pi}{2}$ regardless of $z_{k}$ such that a stable cholesteric helix is propagating in the $z$-direction. Because we partitioned our system into slices (i.e., lattice planes) along the $z$-direction we obtain the local director for each slice as detailed above. Consider now the angle $\psi_{k, k+1}$ between local directors in adjacent slices or planes

$$
\cos \psi_{k, k+1}=\widehat{\boldsymbol{n}}\left(z_{k}\right) \cdot \widehat{\boldsymbol{n}}\left(z_{k+1}\right) \equiv \widehat{\boldsymbol{n}}_{k} \cdot \widehat{\boldsymbol{n}}_{k+1} .
$$

Using the far right-hand side of Equation (24) we can easily solve Equation (25) to obtain

$$
q=\frac{1}{n-1} \frac{1}{\delta_{\mathrm{z}}} \sum_{k=1}^{n-1} \psi_{k, k+1}
$$


where we average over pairs of neighbouring slices (lattice planes) to enhance the statistical accuracy. In Section 4 we will present plots of the ensemble averaged $q$. Because of the uniaxial symmetry of $\widehat{\boldsymbol{n}}$ we determine the correct $\psi_{k, k+1}$ from the expression

$$
\psi_{k, k+1}=\min \left[\psi_{k, k+1}, \pi-\psi_{k, k+1}\right] .
$$

As we explained in Section 2.1.2 the cholesteric helix can be left- or right-handed. Consequently, $q$ can be positive or negative. To determine the correct sign we consider the scalar triple product $\left(\widehat{\boldsymbol{n}}_{k} \cdot \widehat{\boldsymbol{n}}_{k+1}\right)\left(\widehat{\boldsymbol{n}}_{k} \times \widehat{\boldsymbol{n}}_{k+1}\right) \cdot \widehat{\boldsymbol{e}}_{\mathrm{z}}$ [cf., Equation (A6)]. The sign of the previous expression indicates the handedness of the cholesteric helix.

\subsection{A simple theoretical approach to estimate the helical wavenumber}

To analyse our simulation results further we now develop a simple theory that allows us to estimate the helical wavenumber $q$. We devise this theory for the off-lattice model because it is the most general one considered in this work. In the off-lattice model the orientation dependence of the interaction potential is given by

$$
u \propto \varepsilon_{1}\left(\widehat{\boldsymbol{r}}_{12} \cdot \widehat{\boldsymbol{e}}_{\mathrm{z}}\right)^{2}\left(\widehat{\boldsymbol{u}}_{1} \times \widehat{\boldsymbol{u}}_{2}\right) \cdot \widehat{\boldsymbol{r}}_{12}\left(\widehat{\boldsymbol{u}}_{1} \cdot \widehat{\boldsymbol{u}}_{2}\right)+\varepsilon_{2}\left(\widehat{\boldsymbol{u}}_{1} \cdot \widehat{\boldsymbol{u}}_{2}\right)^{2} .
$$

Let us assume that the magnitude of $\varepsilon_{1}$ is sufficiently large so that $\widehat{\boldsymbol{u}}_{i} \rightarrow \widehat{\boldsymbol{u}}_{i}^{\prime}(i=1,2)$ where $\widehat{\boldsymbol{u}}_{i}^{\prime}$ is given by Equation (8) for the special case $\theta_{i}=\frac{\pi}{2}$. In other words, we assume that the orientations of the mesogens are restricted to the $x-y$ plane as in the $\mathcal{S}^{1}$-model. It is then a simple matter to verify that

$$
\begin{aligned}
\widehat{\boldsymbol{u}}_{1} \times \widehat{\boldsymbol{u}}_{2} & =\left(\cos \phi_{1} \sin \phi_{2}-\sin \phi_{1} \cos \phi_{2}\right) \widehat{\boldsymbol{e}}_{\mathrm{z}} \\
\widehat{\boldsymbol{u}}_{1} \cdot \widehat{\boldsymbol{u}}_{2} & =\cos \phi_{1} \cos \phi_{2}+\sin \phi_{1} \sin \phi_{2} .
\end{aligned}
$$

We now express $\widehat{\boldsymbol{r}}_{12}$ in spherical coordinates according to the expression

$$
\widehat{\boldsymbol{r}}_{12}=\left(\begin{array}{c}
\sin \theta \cos \phi \\
\sin \theta \sin \phi \\
\cos \theta
\end{array}\right) \text {. }
$$

With Equations (29) and (30) we can rewrite the right-hand side of Equation (28) as

$$
u \propto-\frac{\varepsilon_{1}}{2} \sin \left[2\left(\phi_{1}-\phi_{2}\right)\right] \cos ^{3} \theta+\frac{\varepsilon_{2}}{2} \cos \left[2\left(\phi_{1}-\phi_{2}\right)\right]+\frac{\varepsilon_{2}}{2} .
$$

Maximising the right-hand side with respect to the angle difference $\phi_{1}-\phi_{2}$ [i.e., minimising $u$ ] we obtain

$$
\tan \left[2\left(\phi_{1}-\phi_{2}\right)\right]=-\frac{\varepsilon_{1}}{\varepsilon_{2}} \cos ^{3} \theta
$$

Within the (open) interval $]-\frac{\pi}{2}, \frac{\pi}{2}[$ the tangent function is bijective. Hence, for 
$\left|\phi_{1}-\phi_{2}\right|<\frac{\pi}{4}$ we can invert the previous equation and obtain

$$
\phi_{1}-\phi_{2}=\frac{1}{2} \arctan \left(-\frac{\varepsilon_{1}}{\varepsilon_{2}} \cos ^{3} \theta\right) \gtreqless 0,
$$

where $\theta \in[0, \pi]$ is the polar angle between $\widehat{\boldsymbol{r}}_{12}$ and the laboratory $z$-axis.

The equality in Equation (33) holds if the cholesteric phase degenerates to an ordinary (perfect) nematic phase; the cholesteric helix is right-handed if $\phi_{1}-\phi_{2}<0$ or left-handed if $\phi_{1}-\phi_{2}>0$ [see Figure 1(a)]. Because the arctangent function on the right-hand side of Equation (33) is point-symmetric it changes sign at $\theta=\frac{\pi}{2}$. This means that even at fixed $\varepsilon_{1} / \varepsilon_{2}, \phi_{1}-\phi_{2}$ changes sign with $\theta$. However, on account of these symmetry properties it is physically nonsensical if $\phi_{1}-\phi_{2}$ changes sign unless the ratio $\varepsilon_{1} / \varepsilon_{2}$ does. This is confirmed by Figure $1(\mathrm{~b})$ where we present a typical "snapshot" of a configuration obtained in our simulations.

To get access to the helical wavenumber, one needs to average the far right-hand side of Equation (33) over the angle $\theta$. Thus, because of the aforementioned symmetry properties of the arctangent function and the range of values on which $\theta$ is defined, some caution is advisable when performing this average.

With the aid of Equation (33) we can therefore define the helical wavenumber $q_{0}$ for our theoretical model as

$$
q_{0} R \equiv \frac{1}{2} \operatorname{sgn}\left(\frac{\varepsilon_{1}}{\varepsilon_{2}}\right) \int_{-1}^{1} \mathrm{~d} x\left|\arctan \left(-\frac{\varepsilon_{1}}{\varepsilon_{2}} x^{3}\right)\right| \tilde{f}(x),
$$

where $R$ is some characteristic length scale (see below), sgn is the signum function, $x \equiv$ $\cos \theta$ and $\widetilde{f}$ is a function describing the distribution of $\widehat{\boldsymbol{r}}_{12}$ with respect to the (positive) $z$-axis. On account of the definition of $\widetilde{f}$ as a distribution function, $\int_{-1}^{1} \mathrm{~d} x \widetilde{f}=1$.

Considering the $\mathcal{S}^{1}$ - or the $\mathcal{S}^{2}$-model we specify $\tilde{f}$ via the ansatz

$$
\widetilde{f}(x) \equiv \frac{1}{2}[\delta(x-1)+\delta(x+1)],
$$

where $\delta$ denotes the Dirac $\delta$-function. This form of $\widetilde{f}$ is obviously motivated by the fact that in both lattice models the chiral interactions are taken into account between a lattice site and its two nearest neighbours in the lattice planes directly above or below. In this case, $\widehat{r}_{12}$ is completely aligned with either the $+z$ - or the $-z$-axis. We can then rewrite Equation (34) as

$$
q_{0} R=\frac{1}{4}\left[\int_{-1}^{0} \mathrm{~d} x \arctan \left(-\frac{\varepsilon_{1}}{\varepsilon_{2}} x^{3}\right) \tilde{f}(x)-\int_{0}^{1} \mathrm{~d} x \arctan \left(-\frac{\varepsilon_{1}}{\varepsilon_{2}} x^{3}\right) \tilde{f}(x)\right]
$$

Because of the form of $\tilde{f}$ introduced in Equation (35) both integrals in this expression can be solved analytically. Thus, we readily arrive at

$$
q_{0} R=\frac{1}{2} \arctan \left(\frac{\varepsilon_{1}}{\varepsilon_{2}}\right)
$$


which has been obtained by Luckhurst et al. [15] by a different route. Assuming $\left|\varepsilon_{1} / \varepsilon_{2}\right| \ll 1$, we can expand $\arctan \left(\varepsilon_{1} / \varepsilon_{2}\right)$ in a Taylor series. Truncating this series and retaining only the leading term we obtain

$$
q_{0} R=\frac{\varepsilon_{1}}{2 \varepsilon_{2}}
$$

which is equivalent to Equation (5.3) of van der Meer et al. [14].
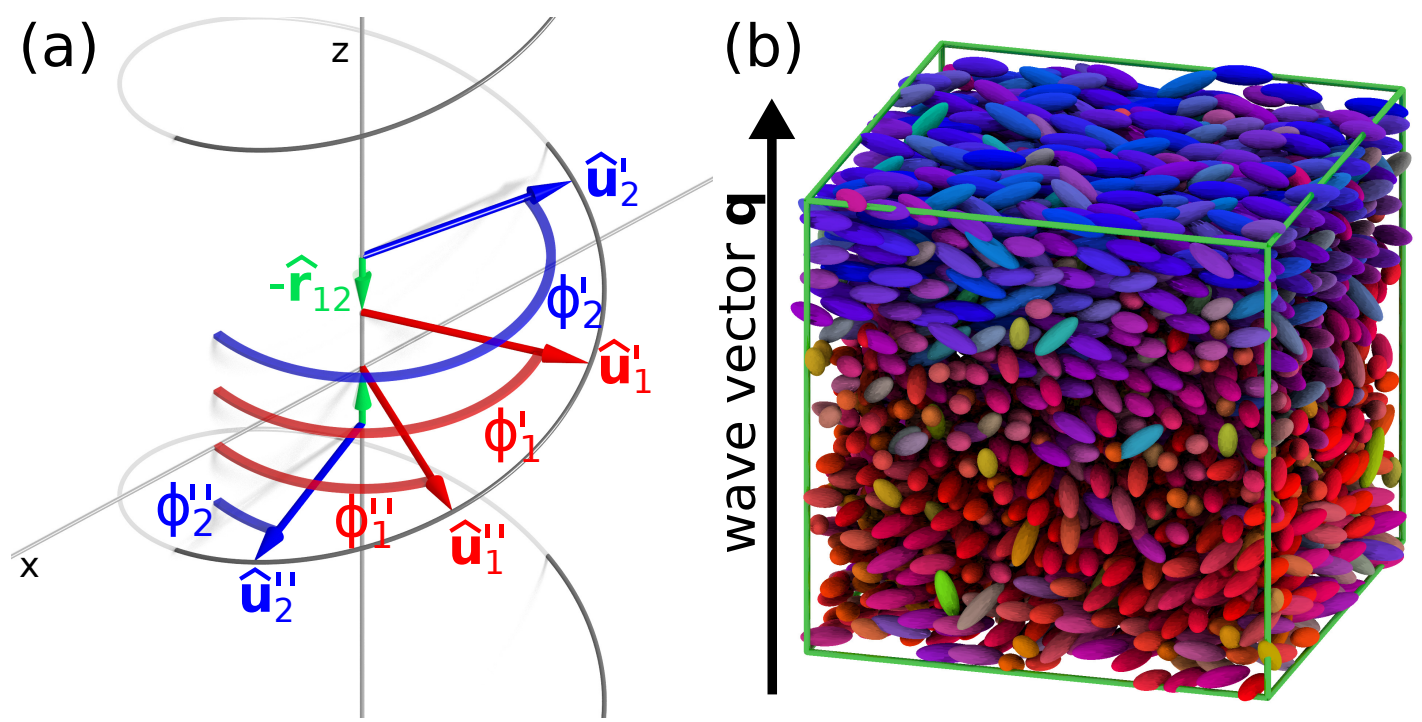

Figure 1. (a) Sketch of a right-handed cholesteric helix evolving in the $z$-direction. Parts of the helix in front of the $x-z$ plane are coloured in black whereas those parts behind the $x-z$ plane are shaded in grey. The orientations of two mesogens are given by $\widehat{\boldsymbol{u}}_{1}^{\prime, \prime \prime}$ and $\widehat{\boldsymbol{u}}_{2}^{\prime, \prime \prime}$, respectively. The arcs indicate the respective angles $\phi_{1}^{\prime \prime \prime}$ and $\phi_{2}^{\prime, \prime \prime}$. The orientation of $\widehat{\boldsymbol{r}}_{12}$ is pointing upward and downward $\left(-\widehat{\boldsymbol{r}}_{12}\right)$ as indicated by the green arrows on the $z$-axis such that the three unit vectors always form a right-handed system. (b) "Snapshot" of a configuration where the mesogens are represented by ellipsoids and coloured according to their orientation. The aspect ratio of the ellipsoids is greatly exaggerated for reasons of visibility.

To derive analogous expressions for $q$ in the off-lattice model one needs to realise that, unlike for the $\mathcal{S}^{1}$ and $\mathcal{S}^{2}$ lattice models, the distribution of $\widehat{\boldsymbol{r}}_{12}$ with respect to the laboratory $z$-axis is not $\delta$-like but isotropic. We have checked that is, in fact, so by computing the distribution function in our MC simulations. Replacing in Equation (34) $\tilde{f}$ by a new distribution function $f=$ const, Equation (34) readily simplifies to

$$
q_{0} R=\int_{0}^{1} \mathrm{~d} x \arctan \left(\frac{\varepsilon_{1}}{\varepsilon_{2}} x^{3}\right),
$$

where we assumed that $\int_{-1}^{0} \mathrm{~d} x f=\int_{0}^{1} \mathrm{~d} x f=1$. This integral can also be solved in closed form. However, this solution is algebraically too involved to be useful from a practical perspective. We therefore compute $q_{0} R$ numerically. Finally, we follow van der Meer et al. [14] and identify $R$ with the nearest-neighbour distance between a pair of mesogens. This quantity is very slightly temperature-dependent. For convenience we shall be ignoring this slight dependence on $T$ and simply use $R / \sigma=1$. 


\section{Results}

\subsection{Numerical details}

For all quantities calculated in our MC simulations we employ the conventional dimensionless (i.e., "reduced") units. A comprehensive compilation of these units can be found in Appendix B of the book by Allen and Tildesley [33]. Simulations for the $\mathcal{S}^{1}$ and $\mathcal{S}^{2}$-model invoke simple-cubic lattices of $N=30 \times 30 \times 30=2.7 \times 10^{4}$ nodes with only nearest-neighbour interactions. The simulations are based upon $3.0 \times 10^{4}$ $\mathrm{MC}$ cycles for equilibration followed by another $7.0 \times 10^{4}$ cycles during which averages are accumulated. An MC cycle consists of $N$ rotations of the mesogens followed by one attempt to change the helical wavenumber at random (see below).

Because the MC simulations of the off-lattice model are computationally much more demanding we employ only smaller systems with $N=3.0 \times 10^{3}$ mesogens. To save computer time as much as possible the interactions between the mesogens are cut off if their centre-of-mass distance exceeds $r_{\mathrm{c}}=3.0$. In addition, we invoke a combination of a link-cell and a Verlet neighbour list [33]. A mesogen is considered to be a neighbour of a reference mesogen if their centres of mass are separated by a distance $r_{\mathrm{N}}=3.5$ or less. The off-lattice simulations are carried out in the isothermal-isobaric ensemble at a constant pressure $P=1.00$.

\subsubsection{The lattice models}

Boundary conditions may give rise to serious problems in computer simulations of chiral bulk systems [34]. This is because the size of a cholesteric helix does not necessarily match the dimensions of the simulation cell in the direction in which this helix evolves. If that is so the helix would be under some artificial stress induced by the boundary conditions. The usual toroidal boundary conditions therefore cannot be applied in this direction. The problem has, of course, been noted before and various clever ways to cure it have been devised [15, 16, 22, 34].

In this work we employ the so-called "spiralling algorithm" devised some time ago by Saslow and coworkers in the context of chiral spin lattices [35, 36]. On account of Equation (12) the cholesteric helix evolves in the $z$-direction of the laboratory reference frame. In that direction the lattice is amended by two virtual layers, say 0 and $n+1$ at the bottom and at the top, respectively. Mesogens located in these virtual layers do not participate in the evolution of the system but only serve to provide suitable boundary conditions.

Initially, the orientations of the $n \times n$ mesogens in the virtual top and bottom layers are set up as follows. Pick an initial value of $q$ at random. The orientations of the mesogens in the bottom and top virtual layers are then obtained by

$$
\begin{aligned}
\widehat{\boldsymbol{u}}_{i}^{(0)} & =\mathbf{R}_{\mathrm{z}}^{n}\left(-q \delta_{\mathrm{z}}\right) \widehat{\boldsymbol{u}}_{i}^{(n)} \\
\widehat{\boldsymbol{u}}_{i}^{(n+1)} & =\mathbf{R}_{\mathrm{z}}^{n}\left(q \delta_{\mathrm{z}}\right) \widehat{\boldsymbol{u}}_{i}^{(1)},
\end{aligned}
$$

for $i=1, \ldots, n \times n$ mesogens in both virtual layers where the upper index refers to the layer,

$$
\mathbf{R}_{\mathrm{z}}(\chi)=\left(\begin{array}{ccc}
\cos \chi & -\sin \chi & 0 \\
\sin \chi & \cos \chi & 0 \\
0 & 0 & 1
\end{array}\right)
$$


describes a rotation around the $z$-axis by an amount $\chi$ and therefore

$$
\begin{aligned}
\mathbf{R}_{\mathrm{z}}^{n}(\chi) & =\underbrace{\mathbf{R}_{\mathrm{z}}(\chi) \mathbf{R}_{\mathrm{z}}(\chi) \ldots \mathbf{R}_{\mathrm{z}}(\chi)}_{n \text { times }} \\
& =\left(\begin{array}{ccc}
\cos (n \chi) & -\sin (n \chi) & 0 \\
\sin (n \chi) & \cos (n \chi) & 0 \\
0 & 0 & 1
\end{array}\right)
\end{aligned}
$$

represents an $n$-fold rotation. The MC algorithm proceeds as follows. We consider each lattice site in a sequential fashion. A mesogen is rotated by a small amount $\delta_{\omega}=$ $\delta_{\omega_{\mathrm{m}}}(1-2 \zeta)$ around one of the three reference axes (selected with equal probability among the three Cartesian axes) where $\delta_{\omega_{\mathrm{m}}}$ is the maximum angle increment for the rotation of a mesogen and $\zeta \in[0,1]$ is a uniformly distributed pseudo-random number. The rotation is accepted on the basis of the standard Metropolis algorithm [33, 37]. Over the course of a simulation $\delta_{\omega_{\mathrm{m}}}$ is adjusted to guarantee an acceptance ratio of $40-60 \%$ of all attempted rotations.

If mesogen $i$ happens to be located in the real lattice planes 1 or $n$ the corresponding mesogen in the respective virtual planes $n+1$ or 0 needs to be updated via Equations (40a) or (40b) prior to applying the Metropolis criterion; if the rotation of mesogen $i$ is rejected so must be the attempted rotation of its virtual counterpart.

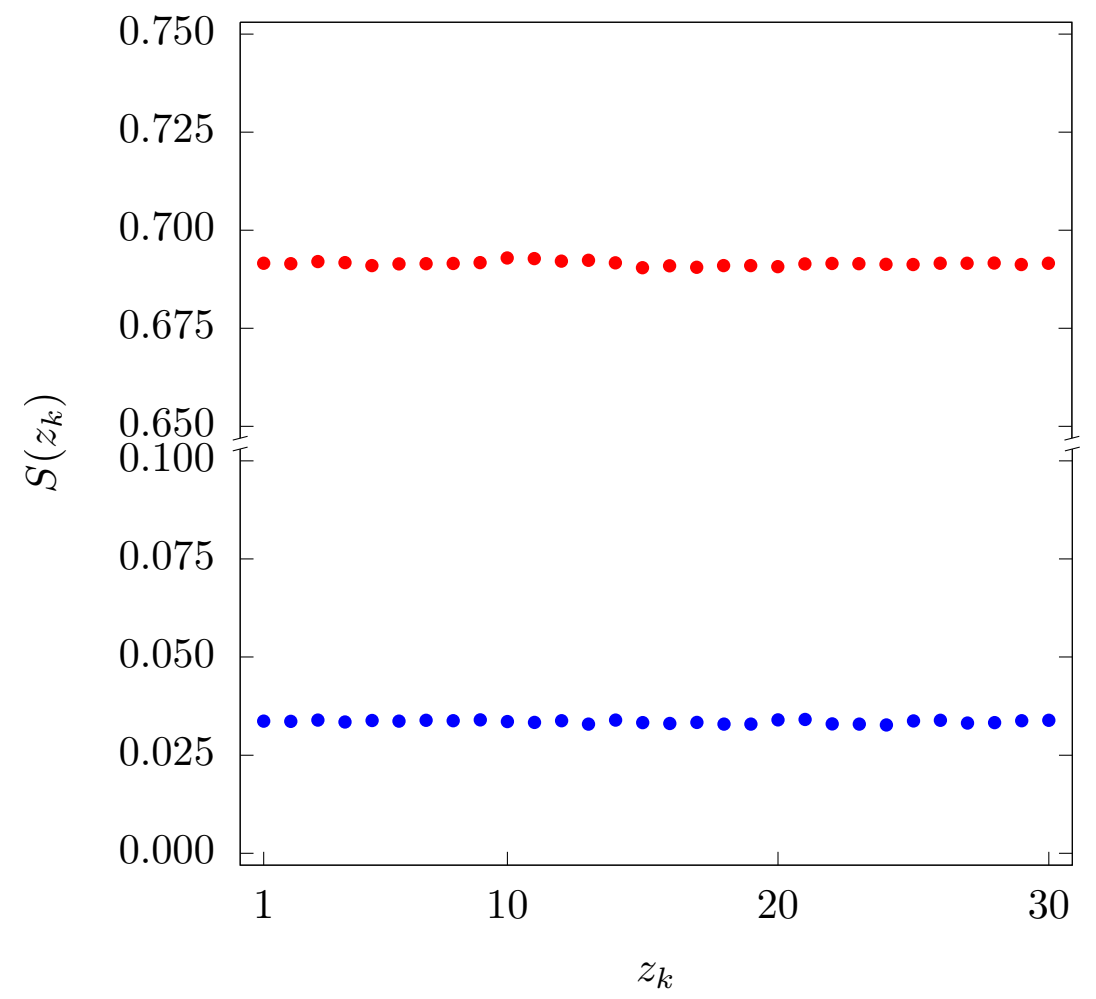

Figure 2. Plot of the local nematic order parameter $S\left(z_{k}\right)(k=1, \ldots, n)$ along the cholesteric helix for $\varepsilon_{1}=0.025$ and $\varepsilon_{2}=0.250 ;(\boldsymbol{\Delta}) T=1.50,(\bullet) T=0.60$.

After all $N$ mesogens have been considered, one attempt is made to randomly change 
$q$ via

$$
\begin{aligned}
q_{\text {new }} & =\delta_{q}(1-2 \zeta) \\
\widehat{\boldsymbol{u}}_{i, \text { new }}^{(k)} & =\mathbf{R}_{\mathrm{z}}^{k}\left(q_{\text {new }} \delta_{\mathrm{z}}\right) \widehat{\boldsymbol{u}}_{i, \text { old }}^{(k)}, \quad k=1, \ldots, n+1, \quad i=1, \ldots, n \times n .
\end{aligned}
$$

Again, the standard Metropolis criterion is applied to decide whether the random change of the helical wavenumber is permissible [33,37]. The ratio of $1: N$ of winding/unwinding the cholesteric helix and rotation attempts of the individual mesogens is chosen because during an attempted change of $q$ the total configurational energy before and after this attempt has to be calculated. This requires the evaluation of $6 \mathrm{~N}$ interactions whereas only 6 interactions need to be considered before and after a rotation of an individual mesogen. The increment $\delta_{q}=3.0 \times 10^{-3} \sigma^{-1}$ is fixed during a simulation. In the $x$ - and $y$-direction the standard toroidal boundary conditions are applied.

To demonstrate that our implementation of the spiralling algorithm works we consider in Figure 2 plots of the local nematic order parameter $S$ [see Equation (18)] for an isotropic and a cholesteric phase. The data are plotted along the $z$-axis, i.e. along the propagation direction of the cholesteric helix. As one can see from the figure the local nematic order is translationally invariant in that direction as it must. Thus, the spiralling algorithm as implemented here does not cause any spurious effects.

In their work, Luckhurst et al. [15] claim that their results are also based upon the spiralling algorithm. However, a comparison with the original algorithm by Saslow and coworkers $[35,36]$ reveals that this is clearly not the case. For instance, the original spiralling algorithm does not require to recalculate $q$ and then use this new $q$ during the generation of the Markov chain. Unfortunately, no rationale for this modification of the original spiralling algorithm is offered by Luckhurst et al. [15].

We have tentatively implemented the algorithm proposed by Luckhurst et al. [15]. Unfortunately, it turned out that in plots analogous to the ones shown in Figure 2, $S$ is not translationally invariant but decayed substantially by about $20 \%$ towards the top and bottom planes of the lattice. From this observation we conclude that the algorithm proposed by Luckhurst et al. [15] is inadequate.

Memmer and Janssen [16] also claim to have compared their algorithm with the spiralling algorithm of Saslow and coworkers [35, 36] and that both give consistent results. However, they give very few details and provide no evidence that this latter statement is, in fact, correct. Instead, the algorithm they propose in place of the spiralling algorithm and which they actually use seems unnecessarily complicated and remains unjustified as well.

\subsubsection{The off-lattice model}

Unlike in the $\mathcal{S}^{1}$ - and $\mathcal{S}^{2}$-model, the off-lattice model does not permit to employ the boundary conditions proposed by Saslow and coworkers $[35,36]$ without amendment. This is because in the off-lattice model the mesogens move along continuous trajectories. Because of this motion and because of the short-range character of $u$ we also need to apply the minimum image convention $[33,37]$. To implement the latter properly we proceed as follows.

Let us begin by introducing scaled coordinates for the interparticle distance via $\boldsymbol{r}_{12}^{\prime}=$ $\boldsymbol{r}_{12} / s$ where $s=n \delta_{\mathrm{z}}$ (see Section 3.1) is the side length of the (cubic) computational cell. We assume that the coordinate system has been chosen such that its origin is at the centre of the computational cell and that $\left(\boldsymbol{r}_{i} \cdot \widehat{\boldsymbol{e}}_{\alpha}\right) s^{-1} \in\left[-\frac{1}{2}, \frac{1}{2}\right](\alpha=x, y, z)$. On 


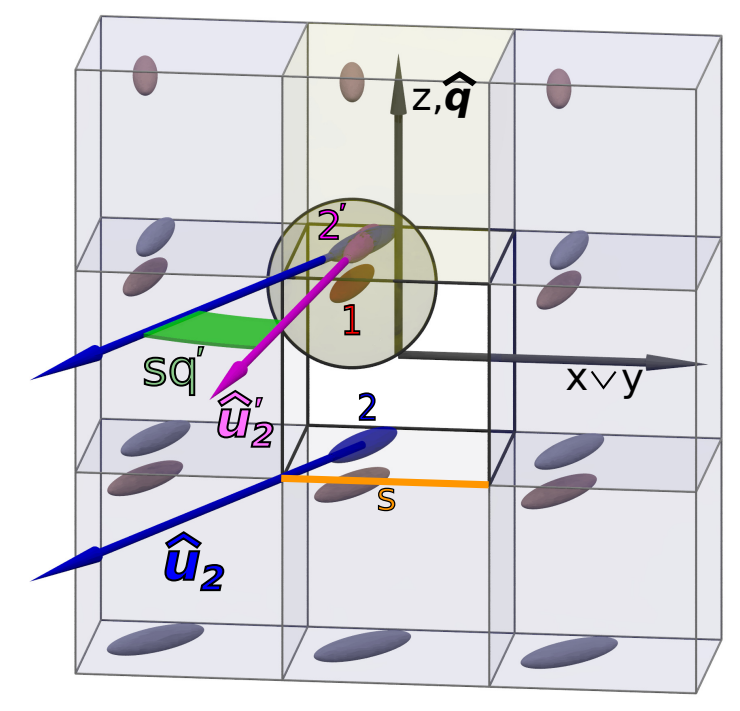

Figure 3. Sketch of the amended spiralling boundary conditions of Saslow and coworkers [35, 36]. The wave vector $\widehat{\boldsymbol{q}}$ is pointing along the $z$-axis of a space-fixed reference frame. The potential cutoff sphere represented by the shaded sphere is centred on mesogen 1 . The $x$ - or $y$-axes are orthogonal to $\widehat{\boldsymbol{q}}$ (see text).

account of Equations (11) and (12) the cholesteric helix evolves in the $z$-direction. This allows us to apply the standard toroidal boundary conditions in the $x$ - and $y$-directions. As we already explained this is in general not possible in the $z$-direction.

To circumvent this problem, let us consider

$$
q^{\prime} \equiv \operatorname{sgn}\left(\boldsymbol{r}_{12}^{\prime} \cdot \widehat{\boldsymbol{e}}_{\mathrm{z}}\right)\left\lfloor\left|\boldsymbol{r}_{12}^{\prime} \cdot \widehat{\boldsymbol{e}}_{\mathrm{z}}\right|+\frac{1}{2}\right\rfloor q,
$$

where $\lfloor x\rfloor \equiv \max \{k \in \mathbb{Z} \mid k \leq x\}$ is the so-called Gaussian bracket returning the largest integer less than or equal to an arbitrary real number $x$. We then obtain the orientation of the minimum image of mesogen 2 via

$$
\widehat{\boldsymbol{u}}_{2}^{\prime}=\mathbf{R}_{\mathrm{z}}\left(s q^{\prime}\right) \widehat{\boldsymbol{u}}_{2},
$$

where $\mathbf{R}_{\mathrm{z}}$ has been introduced in Equation (41).

Once all $N$ mesogens have either been displaced or rotated at random one attempt is made to change the helical wavenumber randomly using Equation (43a). However, on account of the continuous motion of the mesogens in space, Equation (43b) needs to be replaced by

$$
\widehat{\boldsymbol{u}}_{i, \text { new }}=\mathbf{R}_{\mathrm{z}}\left(s q_{i}^{\prime \prime}\right) \widehat{\boldsymbol{u}}_{i, \text { old }}, \quad i=1, \ldots, N,
$$

where $q_{i}^{\prime \prime} \equiv q_{\text {new }} \boldsymbol{r}_{i}^{\prime} \cdot \widehat{\boldsymbol{e}}_{\mathrm{z}}$. As before for the lattice models the change of the helical wavenumber is accepted (or rejected) on the basis of the standard Metropolis criterion $[33,37]$.

The boundary conditions are illustrated by the sketch in Figure 3. The mesogen 1 located at the centre of the potential cutoff sphere does not interact with mesogen 2 but rather with the periodic image $2^{\prime}$ whose orientation is properly altered by the 
angle $s q^{\prime}$ indicated by the arc in Figure 3 . The new orientation $\widehat{\boldsymbol{u}}_{2}^{\prime}$ is then obtained from Equation (45).

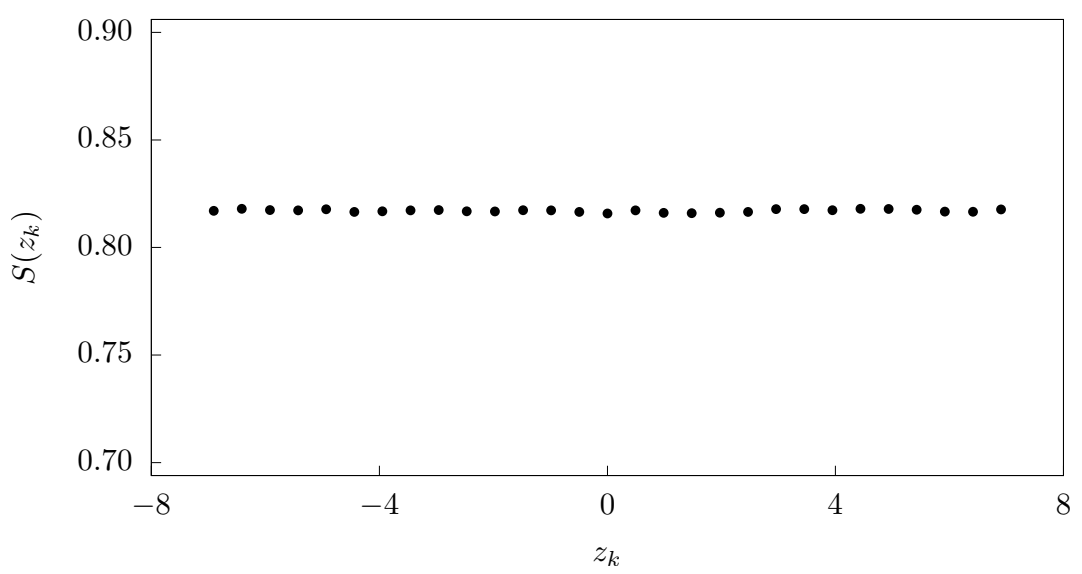

Figure 4. Plot of the local nematic order parameter $S\left(z_{k}\right)$ for the off-lattice model $\left(\delta_{\mathrm{z}}=0.50\right.$, see Section 3$)$ for the coupling constants $\varepsilon_{1}=0.150, \varepsilon_{2}=0.300$, and a temperature $T=0.80$ (cf., Figure 12).

As evidence for the correctness of the amended spiralling boundary conditions we present in Figure 4 a plot of the local nematic order parameter $S$ as a function of the position along the $z$-axis of the laboratory reference frame. These data have been obtained as described in detail in Section 3. As before for the $\mathcal{S}^{2}$-model, the data presented in Figure 4 exhibit no particular dependence on $z$. Therefore, the local nematic order parameter is translationally invariant along the $z$-axis as it must if the amended spiralling boundary conditions are implemented properly.

Moreover, it turned out that in practice it is advantageous to carry out the off-lattice simulations in the isothermal-isobaric ensemble because this reduces the statistical scatter of the computed helical wavenumber significantly. These simulations proceed exactly as described in the books by Frenkel and Smit [37] or Allen and Tildesley [33]. Once all $N$ mesogens have been displaced or rotated at random and the one attempt to change $q$ has been carried out, another attempt is made to alter the volume of the computational cell randomly via

$$
s_{\text {new }}=s_{\text {old }}+\delta_{\mathrm{s}}(1-2 \zeta),
$$

where $\delta_{\mathrm{s}}$ is a small increment that is adjusted during the course of a simulation to ensure that between $40-60 \%$ of all attempted volume changes are accepted according to a modified Metropolis criterion [33, 37]; as in Equation (43a), $\zeta \in[0,1]$ is a pseudorandom number distributed uniformly over that interval. In our version of $\mathrm{MC}$ in the isothermal-isobaric ensemble it is important to make sure that the helical wavenumber remains unaffected by the volume change. We accomplish this by rescaling the helical wavenumber simultaneously according to $q_{\text {new }}=q_{\text {old }} s_{\text {old }} / s_{\text {new }}$ once the volume change is accepted. 


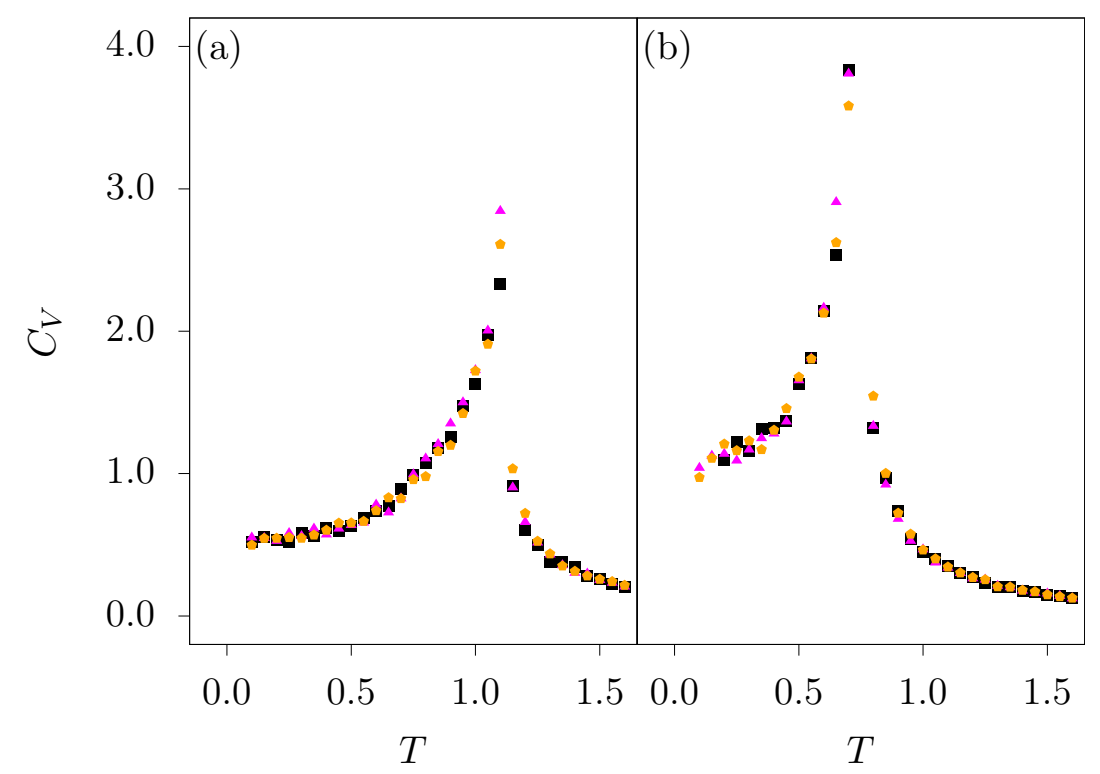

Figure 5. Plots of the isochoric heat capacities $c_{V}$ as functions of the temperature $T$. Part (a) of the figure refers to the $\mathcal{S}^{1}$ - and part (b) to the $\mathcal{S}^{2}$-model (see Section 2.2). Data are presented for $\varepsilon_{1}=0.025(\mathbf{\square})$, $\varepsilon_{1}=0.035(\Delta)$, and $\varepsilon_{1}=0.075$ ( $\Delta$ ) (for all data sets, $\varepsilon_{2}=0.250$ ). The isotropic-cholesteric phase transition occurs at temperatures $T_{\mathrm{ic}} \simeq 1.10$ [part $\left.(\mathrm{a})\right]$ and at $T_{\mathrm{ic}} \simeq 0.75[\operatorname{part}(\mathrm{b})]$, respectively.

\subsection{Lattice Monte Carlo simulations}

To locate the isotropic-cholesteric phase transition we introduce the isochoric specific heat capacity

$$
c_{V}=\frac{1}{k_{B} N T^{2}}\left[\left\langle U^{2}\right\rangle-\langle U\rangle^{2}\right]
$$

where $k_{\mathrm{B}}$ is Boltzmann's constant. The heat capacity is computed from fluctuations of the total configurational potential energy

$$
U=\frac{1}{2} \sum_{i=1}^{N} \sum_{j(i)}^{6} u\left(\boldsymbol{r}_{i j}, \Omega_{i}, \Omega_{j}\right),
$$

because on the lattice the mesogens clearly do not possess any kinetic energy (for a definition of $c_{V}$ for an off-lattice molecular fluid see Equation (3.230) of Ref. [18]). The first summation in Equation (49) is carried out over all sites on the lattice whereas the second one involves the six nearest neighbours of site $i$.

Starting at a sufficiently high $T$ the plots in both parts of Figure 5 indicate that upon lowering $T, c_{V}$ increases monotonically at first. At some characteristic $T=T_{\mathrm{ic}}$ one notices a rather sharp maximum which is indicative of a first-order phase transition from the isotropic to an ordered, cholesteric phase. That this new ordered phase is indeed cholesteric will become clear from other quantities to be discussed below and cannot be inferred directly from the plots in Figure 5 .

Moreover, plots in both parts of Figure 5 clearly show that for the range of chiral coupling strengths considered the value of the characteristic temperature $T_{\mathrm{ic}}$ is insensitive to $\varepsilon_{1}$. However, $T_{\text {ic }}$ is higher for the $\mathcal{S}^{1}$ - compared with the $\mathcal{S}^{2}$-model. This makes physical sense because in the $\mathcal{S}^{1}$ model the mesogens are already forced to lie in the 
$x-y$ plane which makes it easier for them to form a local nematic (i.e., cholesteric) phase.

In addition one notices by comparing the plots in Figures $5(\mathrm{a})$ and $5(\mathrm{~b})$ that in the limits $T \rightarrow 0, c_{V}$ attains different nonzero values. For the $\mathcal{S}^{1}$-model, $\lim _{T \rightarrow 0} c_{V}=$ $\frac{1}{2}$ whereas $\lim _{T \rightarrow 0} c_{V}=1$ for the $\mathcal{S}^{2}$-model. Both limiting values are reported by Memmer and Janssen [16] for the $\mathcal{S}^{1}$-model and some time ago by Chiccoli et al. [38] for an inhomogeneous Lebwohl-Lasher model which is also defined on the $\mathcal{S}^{2}$ sphere. The different values of $c_{V}$ in the limit of vanishing temperature are in line with the equipartition theorem [39] and reflect the different number of orientational degrees of freedom of the mesogens in the $\mathcal{S}^{1}$ - as opposed to the $\mathcal{S}^{2}$-model.

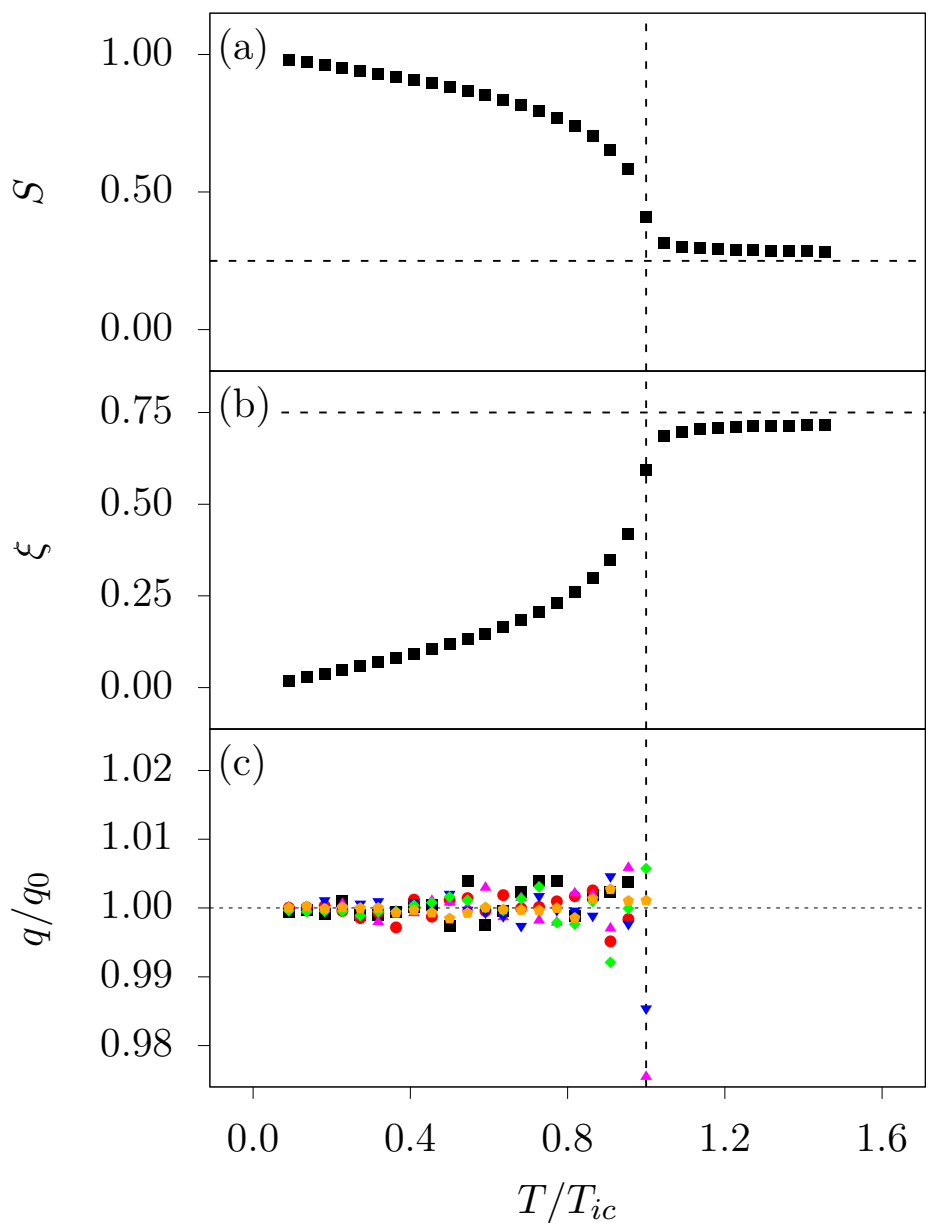

Figure 6. Results for the for the $\mathcal{S}^{1}$-model and $\varepsilon_{2}=0.250$ as functions of the reduced temperature $T / T_{\mathrm{ic}}$ [see Figure 5(a)] ; (a) The nematic order parameter $S$ where the horizontal dashed line corresponds to $S=\frac{1}{4}$ (see text), (b) the biaxiality order parameter $\xi$ where the horizontal dashed line corresponds to $\xi=\frac{3}{4}$ (see text), and (c) the reduced helical wavenumber $q / q_{0}$ [see Equation (37) for a definition of $q_{0}$ ]; $(\boldsymbol{\square}) \varepsilon_{1}=0.025$, (○) $\varepsilon_{1}=0.120,(\boldsymbol{\Delta}) \varepsilon_{1}=0.035,(\boldsymbol{\nabla}) \varepsilon_{1}=0.040,(\diamond) \varepsilon_{1}=0.050,(\Delta) \varepsilon_{1}=0.075$. In part (c) of the figure data are restricted to the cholesteric phase up to $T / T_{\mathrm{ic}} \lesssim 1.00$ demarcated by the vertical dashed line. The dashed horizontal line corresponds to the theoretical value $q=q_{0}$.

In Figure 6 we present plots of various quantities computed for the $\mathcal{S}^{1}$-model as functions of the reduced temperature $T / T_{\mathrm{ic}}$. For example, in Figure 6(a) we present the global nematic order parameter $S$ [see Equation (20)]. In the isotropic phase, i.e., for $T / T_{\text {ic }} \gtrsim 1.00$ we see that $S \simeq \frac{1}{4}$. This is in line with results obtained by Memmer and Janssen [16] [see their Figure 4(a)] and also with data presented by Luckhurst 
et al. [15] where one needs to convert their differently defined order parameter $\bar{T}_{2}$ to our $S$. For $T / T_{\text {ic }}<1.0, S$ increases monotonically with decreasing $T$ indicating the formation of a progressively ordered phase.

To rationalise the nonzero value of $S$ in the isotropic phase let us consider the alignment tensor which can be cast as

$$
Q_{i j}=\frac{3}{2} \int \mathrm{d} \omega u_{i}(\omega) u_{j}(\omega) \alpha(\omega)-\frac{1}{2} \delta_{i j}, \quad i, j=x, y, z,
$$

where $\delta_{i j}$ denotes the Kronecker symbol. On the $\mathcal{S}^{1}$ sphere, $\alpha=\frac{1}{2 \pi} \delta\left(\theta-\frac{\pi}{2}\right)$ in the isotropic phase. Expressing $\widehat{\boldsymbol{u}}$ in spherical coordinates [cf., Equation (8)], it is easy to verify that $\mathbf{Q}$ is diagonal. Thus, with the aid of Equation (18) it is then immediately clear that $\lambda_{+}=\lambda_{0}=\frac{1}{4}$ and $\lambda_{-}=-\frac{1}{2}$. The eigenvector associated with $\lambda_{+}$is pointing in an arbitrary direction of the $x-y$ plane and can be cast as

$$
\widehat{\boldsymbol{n}}_{+}=\frac{1}{\sqrt{a^{2}+b^{2}}}\left(\begin{array}{c}
a \\
b \\
0
\end{array}\right), \quad a, b \in \mathbb{R} .
$$

Keeping in mind that the three eigenvectors of $\mathbf{Q}$ should be pairwise orthogonal to one another we obtain [see Equation (41)]

$$
\widehat{\boldsymbol{n}}_{0}=\mathbf{R}_{\mathrm{z}}( \pm \pi / 2) \widehat{\boldsymbol{n}}_{+}=\frac{1}{\sqrt{a^{2}+b^{2}}}\left(\begin{array}{c}
\mp b \\
\pm a \\
0
\end{array}\right)
$$

Finally, the eigenvalue $\lambda_{-}$of $\mathbf{Q}$ suggests that the associated eigenvector is given by the expression $\widehat{\boldsymbol{n}}_{-}^{\mathrm{T}}=(0,0,1)$ and is therefore orthogonal to the $x-y$ plane. Thus, $\lambda_{+}=\frac{1}{4}$ suggests $S=\frac{1}{4}$ via Equations (19) and (20). The small deviation from this analytic result visible in the plot in Figure 6(a) is caused by the inescapable finiteness of the lattice.

The plot of the biaxiality order parameter $\xi$ presented in Figure $6(\mathrm{~b})$ shows that, similar to $S, \xi$ is nearly constant and relatively large in the isotropic phase, that is for $T / T_{\text {ic }}>1.0$. From the above analysis and with the aid of Equation (21) it is immediately clear that $\xi \simeq \frac{3}{4}$ in the isotropic phase. When the ordered phase begins to form (i.e., at $\left.T / T_{\text {ic }} \lesssim 1.0\right), \xi$ begins to decay monotonically until it reaches its limiting value $\lim _{T \rightarrow 0} \xi=0$ for the perfectly ordered locally nematic structure of the cholesteric phase.

The decay of the biaxiality order parameter $\xi$ in the ordered phase can be understood from the odf shown in Figure 7. As we already pointed out, the odf in the $\mathcal{S}^{1}$-model is centred on $\theta=\frac{\pi}{2}$ and can be represented by a line parallel to the $\varphi$-axis in the isotropic phase. The plot in Figure 7(a) indicates that in the ordered phase for $T / T_{\text {ic }}<1.0$ the odf is nonzero only within a line segment centred on $\varphi=0.0$. The extent of the elongated region shrinks with decreasing temperature. Directly at $T / T_{\mathrm{ic}}=0$ the odf can then be represented by $\alpha=\delta(\phi) \delta\left(\theta-\frac{\pi}{2}\right)$. For this idealised odf and by the same token as before we obtain with the aid of Equation (50) $S=1.0$; because $\lambda_{0}=\lambda_{-}=-\frac{1}{2}$, Equation (21) gives $\xi=0$ at $T / T_{\mathrm{ic}}=0$.

The plot of the helical wavenumber in Figure 6(c) clearly shows that in the $\mathcal{S}^{1}$-model $q$ is independent of $T$ for all chiral coupling constants considered in this work. We therefore confirm earlier findings by Luckhurst et al. [15] and by Memmer and Janssen 


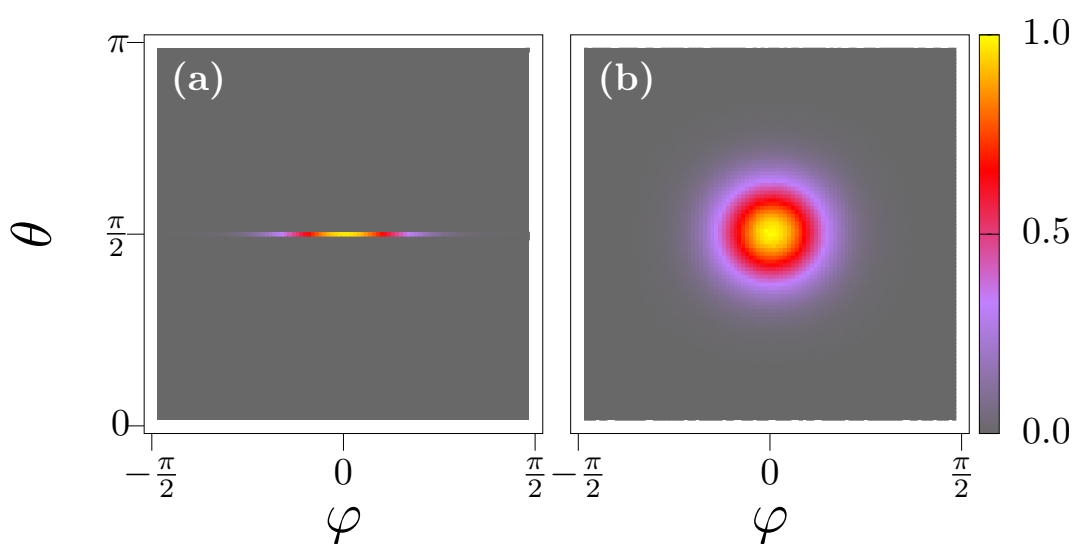

Figure 7. Colour map of the renormalised orientation distribution function $\alpha^{\prime}(\omega)=\alpha(\omega) / \alpha_{\max }$ in the plane of the angles $\theta$ and $\varphi$ where $\alpha_{\max }$ is the maximum value of $\alpha$ (see attached colour bar). The angle $\varphi$ is related to $\phi \in[0,2 \pi]$ in the customary spherical coordinates through the relation $\varphi=\phi-2 \pi \Theta(\phi-\pi)$ where $\Theta$ is the Heaviside function. As a consequence the nematic director $\widehat{\boldsymbol{n}}$ is pointing out of the paper plane; (a) $\mathcal{S}^{1}$ - model, $T / T_{\text {ic }} \simeq 0.91$, (b) $\mathcal{S}^{2}$-model, $T / T_{\text {ic }} \simeq 0.93$. Data in both parts of the figure are obtained for $\varepsilon_{1}=0.025$ and $\varepsilon_{2}=0.250$.

[16] for the $\mathcal{S}^{1}$-model. In addition, the data plotted in Figure 6(c) agree with the prediction of the mean-field theory proposed by van der Meer et al. [14] in its simplest version. This is perhaps not too surprising because the theory by van der Meer et al. [14] mimics the $\mathcal{S}^{1}$-model as well. The magnitude of $q$ is perfectly described by the theoretical value $q_{0}$ given by Equation (37). This is reflected by the fact that regardless of the value of $\varepsilon_{1}, q / q_{0} \simeq 1.0$.

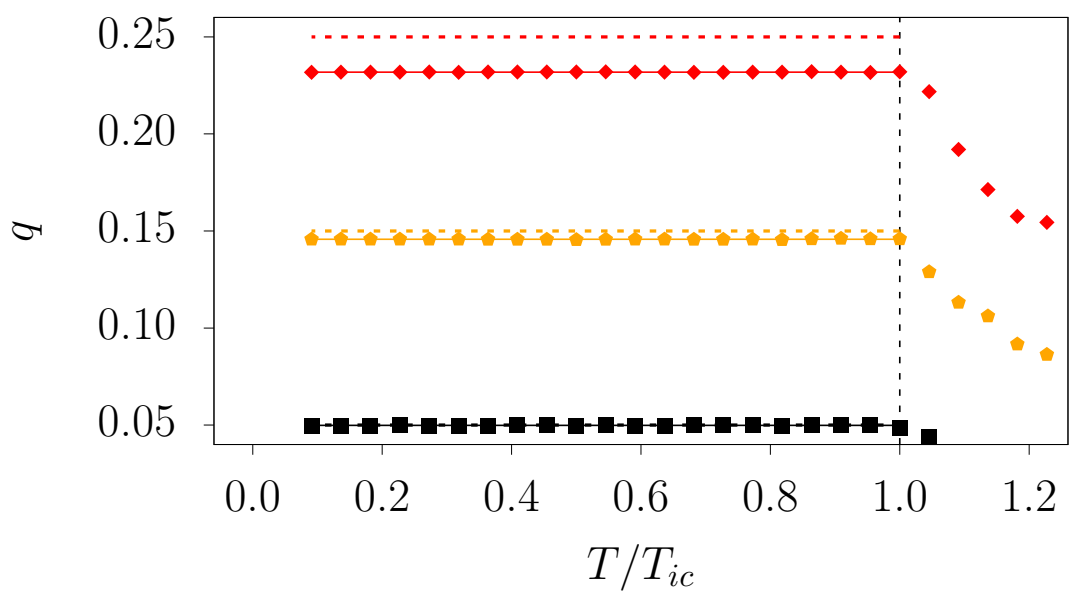

Figure 8. The helical wavenumber $q$ as a function of the reduced temperature $T / T_{\text {ic }}$ for the $\mathcal{S}^{1}$-model; $\varepsilon_{1}=0.025,(\diamond) \varepsilon_{1}=0.075,(\diamond) \varepsilon_{1}=0.125$ (for all data sets, $\varepsilon_{2}=0.250$ ). Solid lines are the theoretical predictions $q_{0}$ using Equation (37) whereas dashed lines correspond to the approximate expression given in Equation (38).

As we already mentioned above, a key ingredient of the mean-field theory of van der Meer et al. is the restriction to small helical wavenumbers. To test the validity of this so-called small-angle approximation we plot in Figure 8 the helical wavenumber for the $\mathcal{S}^{1}$-model from our MC simulations together with the theoretical predictions of Luckhurst et al. [15] [see Equation (37)] and van der Meer et al. [14] [see Equation (38)].

Not unexpectedly, both theoretical results agree nicely with the data from our computer simulations for small ratios of $\varepsilon_{1} / \varepsilon_{2}$. The deviation between the mean-field results 
and the MC simulations increases with an increasing ratio of the coupling constants indicating the beginning failure of the small-angle approximation. On the contrary, Equation (37) works quite well for all the values of $\varepsilon_{1} / \varepsilon_{2}$ considered in Figure 8.

Another feature illustrated by the plots presented in Figure 8 is a non-vanishing $q$ even above the isotropic-cholesteric phase transition. Ideally, $q$ should cease to exist for all $T$ above the transition temperature. That this is not so can be linked to the inevitable finite system size which stabilises vestiges of the helical structure over a finite range of temperatures above the isotropic-cholesteric phase transition.

The same monotonic decay of $q$ above the phase transition has been noted by Luckhurst et al. [15] with an explanation along the same lines. In the paper by Memmer and Janssen a similar decay of $q$ with increasing temperature above the isotropic-cholesteric phase transition was reported but remains unexplained by these authors (see Figure 6 in Ref. [16]). However, because in the present work we are not interested per se in finite-size effects in the isotropic phase, no attempts were made to study the described system-size effects in depth.

While the agreement between our results and the corresponding ones obtained by Luckhurst et al. [15] and by Memmer and Janssen [16] may seem rather gratifying at first, it really does point to a deficiency of the $\mathcal{S}^{1}$-model. As we showed in Section 3.3, Equation (37) is obtained by minimising Equation (31) with respect to $\phi_{1}-\phi_{2}$ (for the special case of $\theta=0$ or $\pi$ on the lattice). Thus, Equation (37) completely ignores entropic effects. The perfect agreement of the theoretical expression with the MC data for the $\mathcal{S}^{1}$-model reveals that this must be a model-inherent feature that renders the $\mathcal{S}^{1}$-model unrealistic.

For the same values of the coupling constant $\varepsilon_{1}$ we also performed MC simulations for the $\mathcal{S}^{2}$-model. In this case, $\alpha=\frac{1}{4 \pi}$ in the isotropic phase and one can easily verify that $\mathbf{Q}=\mathbf{0}$ from Equation (50) where $\mathbf{0}$ is the zero tensor leading to $S=0$. The corresponding plot of $S$ in Figure 9(a) exhibits rather small positive values instead. This is because of a well-understood system-size effect that one may employ in a finitesize scaling approach to locate the phase transition as was demonstrated for a nematic liquid crystal by Greschek and Schoen [40]. At $T / T_{\text {ic }} \simeq 1.0$ an isotropic-cholesteric phase transition occurs and $S$ rises monotonically with decreasing temperature towards the limiting value $\lim _{T \rightarrow 0} S=1$. The curve shown in Figure 9(a) appears to by slightly rounded due to this finite-size effect.

Whereas the overall temperature dependence of $S$ is the same as in the $\mathcal{S}^{1}$-model, the underlying odf turns out to be markedly different as the plot in Figure 7(b) reveals. On account of the two degrees of freedom characterising the $\mathcal{S}^{2}$-model the odf in the ordered phase consists of a centrosymmetric spot centered on $\varphi=0$ and $\theta=\frac{\pi}{2}$. As $T$ decreases, the odf becomes increasingly localised at these values and can eventually be described by the expression $\alpha=\delta(\varphi) \delta\left(\theta-\frac{\pi}{2}\right)$. In other words, the centrosymmetric odf shown in Figure 7(b) shrinks to a point as for the $\mathcal{S}^{1}$-model. This idealised odf is obtained in the limit of vanishing temperature.

Compared with the $\mathcal{S}^{1}$-model the biaxiality order parameter $\xi$ is relatively small here for all temperatures as the plot in Figure 9(b) indicates. It rises with decreasing $T$ towards the isotropic-cholesteric phase transition at $T / T_{\mathrm{ic}} \simeq 1.0$ and then declines monotonically towards $T \rightarrow 0$. A comparison with the plot in Figure 6(b) reveals that the biaxiality order parameter at $T / T_{\text {ic }} \simeq 1.0$ exceeds the one presented in Figure $9(\mathrm{~b})$ by about an order of magnitude due to the unphysical restriction of the orientations of the mesogens to the $x-y$ plane in the $\mathcal{S}^{1}$-model.

The most significant difference between the $\mathcal{S}^{1}$ - and the $\mathcal{S}^{2}$-models concerns the impact of the temperature on the helical wavenumber. The plots in Figure 9(c) exhibit 


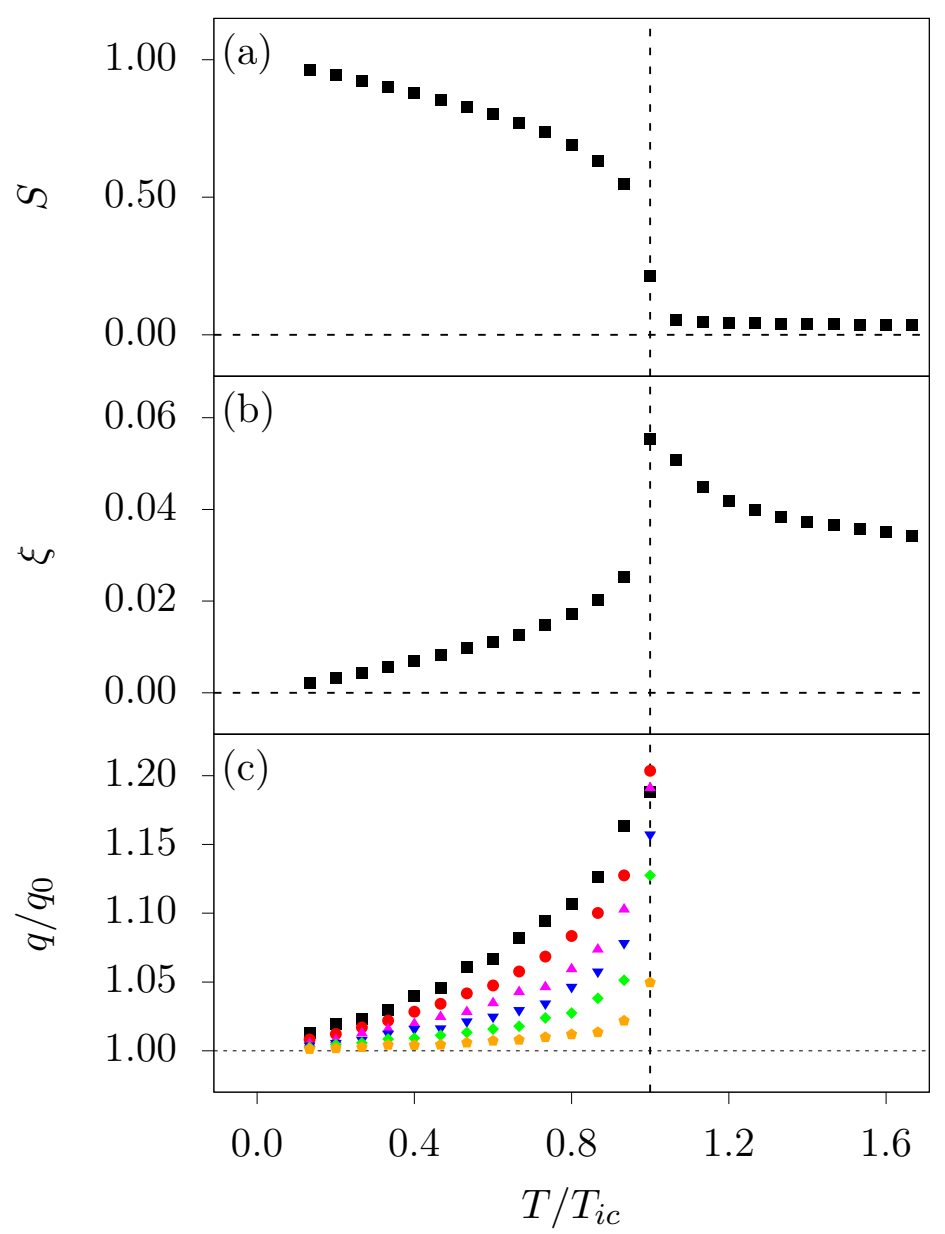

Figure 9. As Figure 6, but for the $\mathcal{S}^{2}$-model. Notice the different scale used for the ordinate in parts (b) and (c) in both figures. The dashed horizontal line in part (c) corresponds to $q=q_{0}$ [see Equation (37)].

a clear tendency for $q$ to increase with increasing $T$ in the cholesteric phase. In general, the smaller $\varepsilon_{1}$ the more pronounced is the increase of $q$ with $T$; the deviation from the theoretical value $q_{0}$ can rise up to about $17 \%$ just below the isotropic-cholesteric phase transitions as entries in Table 1 indicate. This is in line with one's physical intuition because for a weaker chiral interaction between the mesogens their tendency to "escape" to the third dimension (i.e., out of the $x-y$ plane) is enhanced. In other words, the orientational entropy of the mesogens is larger the smaller $\varepsilon_{1}$ is. Notice also that for all entries in Table $1, p$ exceeds the number of lattice planes $n=30$ by more than a factor of 3 at most. This would not be possible had one used the unsuited toroidal boundary also in the propagation direction of the cholesteric helix.

Moreover, the deviation between the MC data plotted in Figure 9(c) from $q / q_{0}=1$ indicates that unlike for the $\mathcal{S}^{1}$-model, Equation (37) works less well. According to our reasoning above this is because in the $\mathcal{S}^{2}$-model entropic effects contribute more substantially; this is because the orientations of the mesogens are not restricted to the circumference of the unit circle. As a consequence, the MC data for the ratio $q / q_{0}$ in Figure 9(c) increase in the cholesteric phase with increasing $T$ towards the isotropiccholesteric phase transition.

The findings based upon the plots shown in Figure 9 are corroborated further by the plot of the order parameter $S_{\theta}$ shown in Figure 10. At temperatures above that 
Table 1. The helical wavenumber $q$ and the associated pitch $p$ for various coupling strengths $\varepsilon_{1}$ in the $\mathcal{S}^{2}$-model $\left[\varepsilon_{2}=0.25\right.$, see Equations (12) and (13)]. All entries in the Table correspond to a thermodynamic state point $T / T_{\mathrm{ic}} \simeq 0.93$ slightly below the temperature of the isotropic-cholesteric phase transition [see Figure 9(c)].

\begin{tabular}{ccccr}
\hline$\varepsilon_{1} / \varepsilon_{2}$ & $q_{0}$ [see Equation $\left.(37)\right]$ & $q$ [see Equation $(26)]$ & $q / q_{0}$ & $p=2 \pi / q^{\mathrm{a}}$ \\
\hline 0.100 & 0.0498 & 0.058 & 1.17 & 108.33 \\
0.120 & 0.0597 & 0.067 & 1.12 & 93.78 \\
0.140 & 0.0695 & 0.077 & 1.11 & 81.60 \\
0.160 & 0.0793 & 0.086 & 1.08 & 73.06 \\
0.180 & 0.0890 & 0.095 & 1.07 & 66.16 \\
0.200 & 0.0987 & 0.104 & 1.05 & 60.42 \\
0.300 & 0.1457 & 0.149 & 1.02 & 42.17 \\
\hline
\end{tabular}

${ }^{\text {a }}$ In units of the lattice constant $\sigma$.

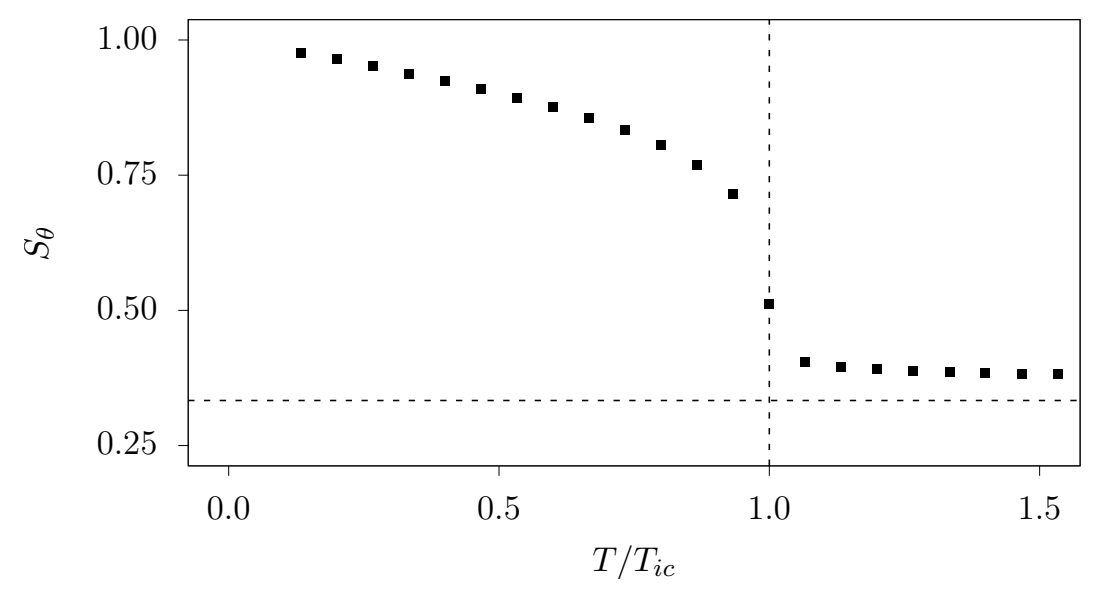

Figure 10. Plot of the order parameter $S_{\theta}$ as a function of the reduced temperature $T / T_{\text {ic }}$ [see Equation (22)]. The dashed horizontal line corresponds to the limiting value $S_{\theta}=\frac{1}{3}$ calculated analytically for the isotropic phase [30]. Results are shown for $\varepsilon_{1}=0.100$ and $\varepsilon_{2}=0.250$.

of the isotropic-cholesteric phase transition $S_{\theta}$ exceeds slightly the theoretical value $S_{\theta}=\frac{1}{3}$ for the isotropic phase. The latter can be obtained analytically as detailed in Ref. [30]. This indicates that in the isotropic phase the tips of the orientation vectors are distributed uniformly on the $\mathcal{S}^{2}$ sphere. As its counterpart $S$ plotted in Figure 9(a), $S_{\theta}$ rises quite abruptly in the vicinity of the isotropic-cholesteric phase transition at $T / T_{\text {ic }}=1$.

For temperatures below that of the isotropic-cholesteric phase transition (i.e., for $\left.T / T_{\text {ic }}<1\right), S_{\theta}$ rises monotonically with decreasing $T$ towards its limiting value $S_{\theta}=1$ to be reached in the limit of vanishing $T$. On account of its definition in Equation (22) the deviation of $S_{\theta}$ from its limiting value is a measure of the deviation of the $\mathcal{S}^{2}$ from the $\mathcal{S}^{1}$-model, the two becoming identical only at $T=0$.

\subsection{Off-lattice Monte Carlo simulations}

Similar to the lattice models we begin the discussion of the results for the off-lattice model by presenting in Figure 11 data for the isobaric specific heat capacity defined as

$$
c_{\mathrm{P}}=\frac{5}{2} k_{B}+\frac{1}{k_{B} N T^{2}}\left[\left\langle\mathcal{H}^{2}\right\rangle-\langle\mathcal{H}\rangle^{2}\right]
$$




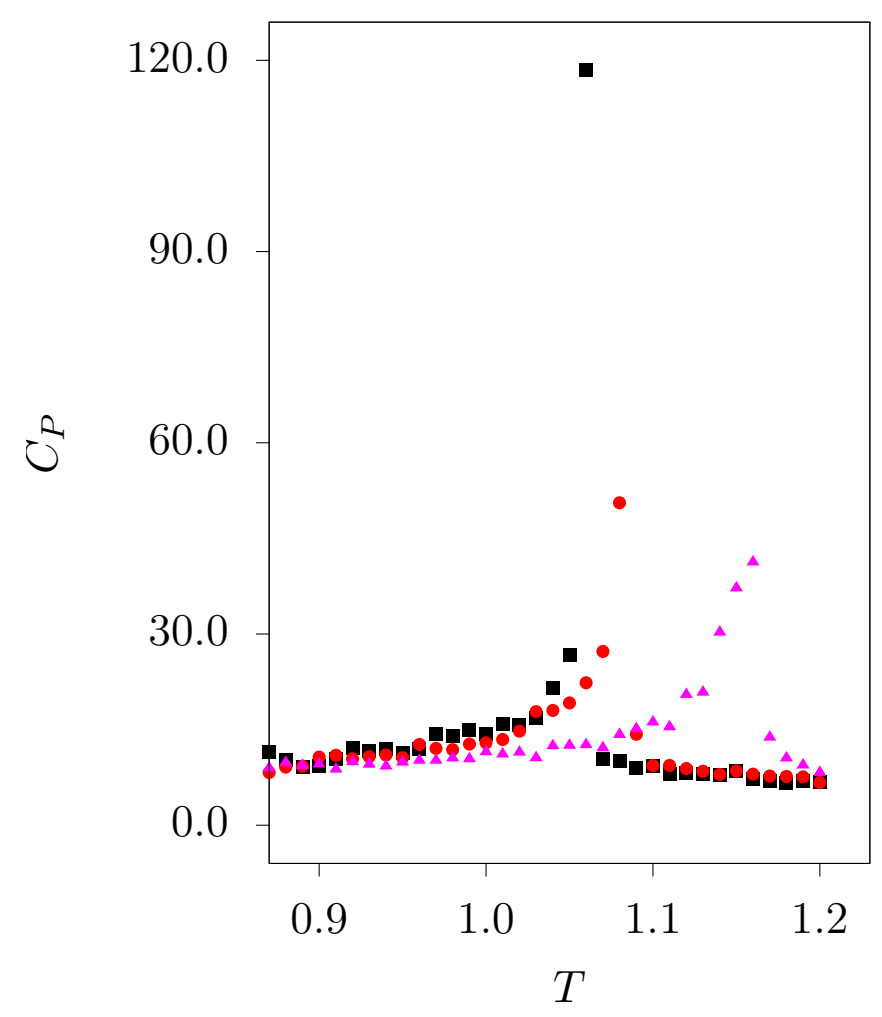

Figure 11. As Figure 5, but for the isobaric heat capacity; $(\boldsymbol{\square}) \varepsilon_{1}=0.150, T_{\mathrm{ic}}=1.06,(\bullet), \varepsilon_{1}=0.300$, $T_{\mathrm{ic}}=1.08,(\boldsymbol{\Delta}) \varepsilon_{1}=0.600, T_{\mathrm{ic}}=1.16$. In all three cases $\varepsilon_{2}=0.300$.

where

$$
\mathcal{H} \equiv \sum_{i=1}^{N-1} \sum_{j=i+1}^{N} u\left(\boldsymbol{r}_{i j}, \omega_{i}, \omega_{j}\right)+P V
$$

is the instantaneous enthalpy of a configuration in the isothermal-isobaric ensemble at constant pressure $P$ and a variable volume $V$ of the computational cell.

Similar to Figure 5, the plots of $c_{\mathrm{P}}$ in Figure 11 show characteristics resembling qualitatively those observed in the plots of $c_{\mathrm{V}}$. For instance, $c_{\mathrm{P}}$ exhibits a sharp maximum at the cholesteric-isotropic transition temperature $T_{\text {ic }}$, with slightly higher values in the cholesteric phase than in the isotropic phase. However, the curves plotted in Figure 11 differ from their counterparts presented in Figure 5 rather markedly. For example, the peak positions in Figure 11 depend on the coupling constants and shift to larger $T$ the larger $\varepsilon_{1} / \varepsilon_{2}$ becomes. This reflects the greater ease with which a cholesteric phase can form at stronger coupling of the chiral contributions to the intermolecular potential.

There is also a lower bound for the temperature range for which the MC simulations in the off-lattice model are technically feasible. This is because on account of the mobility of the mesogens in the off-lattice model they form glassy states if their kinetic energy becomes too small. As we have tested, the thermodynamic states considered here should be safely above the glass-forming regime. This is concluded from the fact that the radial pair correlation function is still that of an ordinary liquid without any other salient features.

In Figure 12(a) we present plots of the local nematic order parameter $S$ as a function of the temperature $T$ for the off-lattice model. As one might have guessed $S$ increases 


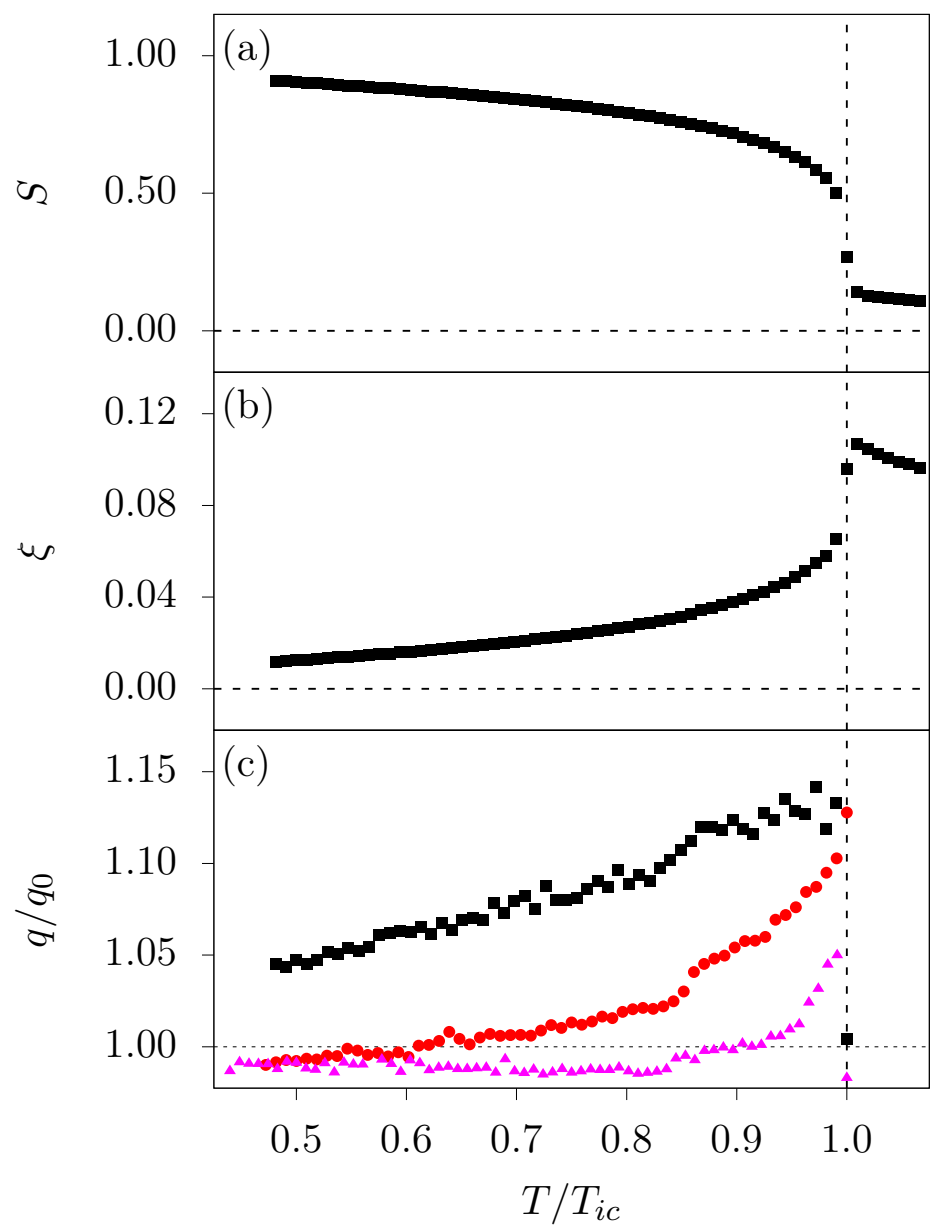

Figure 12. As Figure 6, but for the off-lattice model; $(\mathbf{\square}) \varepsilon_{1}=0.150,(\boldsymbol{O}) \varepsilon_{1}=0.300,(\boldsymbol{\Delta}) \varepsilon_{1}=0.600$. For all data shown, $\varepsilon_{2}=0.300$. In all three parts of the figure the dashed vertical line indicates the isotropic-cholesteric phase transition. In parts (a) and (b) the dashed horizontal line corresponds to the limiting value in a perfect isotropic liquid and a locally perfect uniaxial nematic phase, respectively; in part (c) the dashed horizontal represents the theoretical value $q=q_{0}$ [see Equation (39)].

rather abruptly at $T \simeq T_{\text {ic }}$ and then approaches unity monotonically with decreasing $T$. In the isotropic phase $S$ decreases with increasing $T$ towards zero but will not quite reach that value on account of finite-size effects as before for the $\mathcal{S}^{2}$-model [40]. A comparison with the corresponding plot in Figure 9(a) shows that in this latter case $S$ is indeed much smaller for a lattice of $2.7 \times 10^{4}$ sites as opposed to the off-lattice model comprising only $3.0 \times 10^{3}$ mesogens [see Figure 12(a)].

These results are also reflected by the biaxiality order parameter plotted in Figure 12(b) across the isotropic cholesteric phase transition. It is about a factor of two higher than its counterpart in the $\mathcal{S}^{2}$-model as a comparison with the plot in Figure 9(b) reveals. This is due to the same finite-size effect just mentioned. In the cholesteric phase (i.e., for $T / T_{\text {ic }} \lesssim 1.0$ ), $\xi$ in Figure $12(\mathrm{~b})$ decays towards $\xi \simeq 0.0$ the more ordered the cholesteric phase becomes. Nevertheless it needs to be stressed that apart from the system-size effect the plots in Figures 12(a) and 12(b) are strikingly similar to the corresponding ones for the $\mathcal{S}^{2}$ lattice model shown in Figures 9 (a) and 9 (b). A very similar temperature dependence of the biaxiality order parameter is reported in Ref. [41] in both NMR experiments and a mean-field model of the Helmholtz free energy for cholesteric fluids. 
This holds also for plots of the helical wavenumber $q$ for the off-lattice model shown in Figure 12(c). Because the discrete nature of the $\mathcal{S}^{2}$-model tends to stabilise cholesteric helices much more than is the case in the continuous off-lattice model, the data shown in Figure 9(c) exhibit less statistical scatter than the corresponding ones in Figure 12(c). Nevertheless, the trend is the same. Clearly, the plots in Figure 12(c) reveal that

(1) the helical wavenumber $q$ is temperature dependent and increases towards the isotropic-cholesteric phase transition

(2) the deviation from the theoretical value $q_{0}$ computed via Equation (39) increases with decreasing coupling constant $\varepsilon_{1}$.

However, the reader should appreciate that the thermodynamic state space for the off-lattice model is much more restricted than that of the $\mathcal{S}^{2}$-model. This is again due to the discrete nature of the lattice on which the latter is based. For instance, as we verified the scatter of points for $q / q_{0}$ becomes inacceptably large if one would use similar values for the ratio of coupling constants $\varepsilon_{1} / \varepsilon_{2}$. Consequently, there is an upper bound for the helical pitch of about $p \approx 60$ estimated for $\varepsilon_{1} / \varepsilon_{2}=0.50$ and close to the isotropic-cholesteric phase transition (i.e., for $T / T_{\mathrm{ic}} \simeq 0.991$ ). The increasing statistical noise with decreasing values of the chiral coupling constant are ascribed to a simultaneous decreasing instability of the cholesteric helix in the off-lattice model. On the contrary, in the $\mathcal{S}^{2}$-model the cholesteric helix is stabilised because of the discrete character of the lattice.

Similarly, there also exists an upper bound for the ratio $\varepsilon_{1} / \varepsilon_{2}$ at a given value of $\varepsilon_{2}$. This upper bound is due to the fact that with increasing $\varepsilon_{1}$ the pitch of the cholesteric helix shrinks until it can no longer be accommodated because in the off-lattice model the mesogens also possess translational degrees of freedom that also tend to destabilise the cholesteric helix.

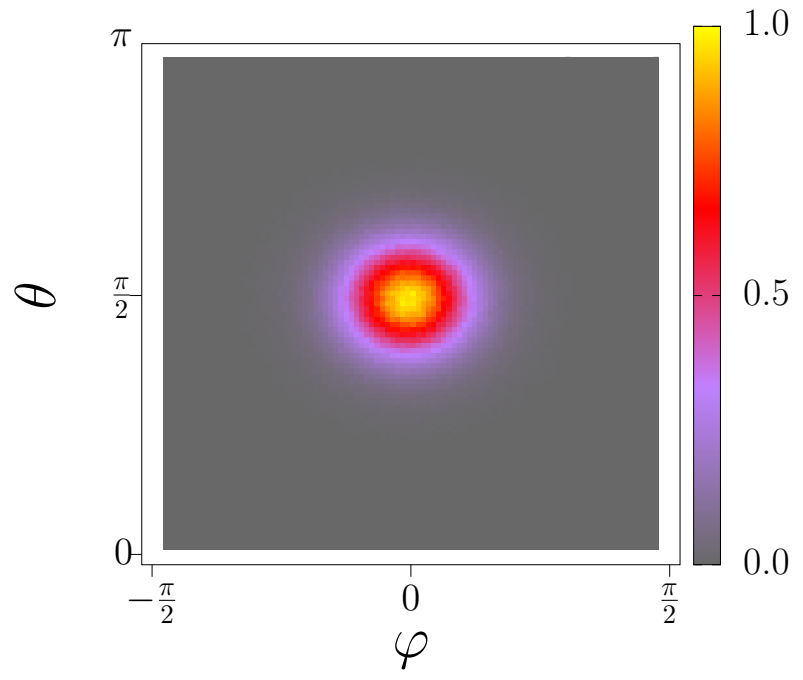

Figure 13. As Figure 7, but for the off-lattice model for $T / T_{\mathrm{ic}} \simeq 0.75$ and $\varepsilon_{1}=0.150$.

Last but not least it is also instructive to analyse the orientational structure of the cholesteric phase via the odf. As before the odf is computed with respect to a local director $\widehat{\boldsymbol{n}}$ that is rotating as one moves between neighbouring slices. The so obtained odf's are then averaged over slices similar to the lattice models where, of course, the averaging is performed over lattice planes. The plot of the odf for the off-lattice model presented in Figure 13 bears a lot of similarity with the one shown in Figure 7(b) for 
the $\mathcal{S}^{2}$-model in that it is (nearly) centrosymmetric in the plane of the angles $\theta$ and $\varphi$. This is again evidence that the ordered phase is indeed cholesteric.

However, closer scrutiny reveals that there is a minute deviation from the perfect centrosymmetry in the plot in Figure 13. The central bright spot in Figure 13 is very slightly elongated along the $\varphi$-axis. This is because the thickness of the slices is finite unlike in the $\mathcal{S}^{2}$-model where the lattice planes across which the odf is evaluated are infinitely thin. Hence, if one enters a slice in the off-lattice model from one side and then moves along the $z$-direction until one reaches its end the director is not entirely constant but rotates around the $z$-axis by a very small amount. This slight rotation is responsible for the small deformation of the odf visible in Figure 13.

\section{Discussion and conclusions}

We carry out MC simulations to investigate the properties of cholesteric phases in simple models of chiral liquid crystals. Two types of models are investigated. In one of these the positions of the mesogens are restricted to the sites of a simple-cubic lattice. The other one is an off-lattice model where the mesogens can freely move in space along continuous trajectories.

On the lattice, the mesogens possess only orientational degrees of freedom. We consider two different lattice models in this work. In the first one the orientations of the mesogens are defined on the $\mathcal{S}^{1}$ sphere. Hence, the tips of the unit vectors describing the orientations of the mesogens lie somewhere on the circumference of the unit circle. For obvious reasons, we refer to this model as the " $\mathcal{S}^{1}$-model". In the analogous $\mathcal{S}^{2}$-model the tips of the orientation vectors are points on the surface of the unit sphere.

Clearly, the $\mathcal{S}^{1}$-model differs from the $\mathcal{S}^{2}$-model in the number of degrees of freedom the mesogens possess. Whereas one angle suffices to specify the orientations of the mesogens in the $\mathcal{S}^{1}$-model, two such angles are required in the $\mathcal{S}^{2}$-model. Thus, the $\mathcal{S}^{1}$-model is more restrictive than the $\mathcal{S}^{2}$-model. The different degrees of freedom have consequences for the physics of both models. For example, in line with the equipartition theorem and in the limit of vanishing temperature the isochoric specific heat $c_{\mathrm{V}}=\frac{1}{2}$ in the $\mathcal{S}^{1}$-model whereas $c_{\mathrm{V}}=1$ in the $\mathcal{S}^{2}$-model and in that same limit.

Another quantity that differs markedly between both versions of the lattice model is the odf. To compute the odf we employ as a basis the eigenvectors of the alignment tensor Q. In a space-fixed reference frame these basis vectors rotate as one moves along the $z$-axis, that is in the direction of the helical axis. One thus anticipates a centrosymmetric odf characteristic of a (locally) nematic phase of uniaxial symmetry.

In the ordered phase the odf in the $\mathcal{S}^{1}$-model turns out to be an (infinitely) thin line along the $\varphi$-axis centered on $\theta=\frac{\pi}{2}$ which reflects the restriction of the orientations of the mesogens to the unit circle. Instead, in the $\mathcal{S}^{2}$-model the odf can be represented by a spot of centrosymmetry centred on $\theta=\frac{\pi}{2}$ and $\varphi=0$ as one would expect.

In the context of cholesteric phases a key quantity is the helical wavenumber $q$ (or the pitch $p$ ) of the cholesteric helix. The mean-field theory by van der Meer et al. [14] showed that for a model akin to the $\mathcal{S}^{1}$ lattice model, $q$ is independent of temperature in the simple version of their theory. Prompted by the observation that experimentally $q$ is generally not independent of $T$, van der Meer et al. [14] proposed a more sophisticated version of their mean-field theory in which such a temperature dependence would arise through various order parameters.

To remain mathematically tractable the mean-field theory is restricted to small helical wavenumbers $q R \lesssim \mathcal{O}\left(10^{-2}\right)$. This allows one to employ expansions in terms 
of $q R$ truncated after the first nontrivial one. An immediate consequence is that the expression for the mean-field free energy derived by van der Meer et al. [14] does not permit one to identify those values of $q$ for which the cholesteric helix would be thermodynamically stable. Here we show that on account of that small-angle approximation the mean-field theory substantially overestimates $q$ for coupling-constant ratios $\varepsilon_{1} / \varepsilon_{2} \gtrsim 0.30$.

The MC simulations by Luckhurst et al. [15] and by Memmer and Janssen [16] employed the $\mathcal{S}^{1}$-model and the helical wavenumber turned out to be temperatureindependent in line with the simple version of the mean-field theory [14]. In the present work we confirm these earlier findings. However, employing the $\mathcal{S}^{2}$-model we demonstrate here that the absence of a temperature dependence of $q$ is merely a consequence of the rather severe and unphysical restriction of the orientations to the unit circle assumed in the $\mathcal{S}^{1}$-model.

In the $\mathcal{S}^{2}$-model and at relatively low values of the chiral coupling constant $\varepsilon_{1}$ the temperature dependence of $q$ turns out to be quite substantial approaching the theoretical limit only for vanishing temperatures, i.e. as $T \rightarrow 0$. The agreement with the temperature-independent theoretical value $q_{0}$ becomes better even at nonzero $T$ if the chiral coupling is sufficiently strong. This is because in that limit the assumption that the orientations of the molecules are restricted to the unit circle becomes more justified.

However, regardless of whether the $\mathcal{S}^{1}$ - or the $\mathcal{S}^{2}$-model are considered they both lack physical realism with respect to a typical soft-matter system such as a liquid crystal. For example, density or pressure do not enter the thermodynamics of lattice models of the present kind in any straightforward fashion. Thus, we also consider an off-lattice version of our model system where this is not a problem. Carrying out the simulations for the off-lattice model in the isothermal-isobaric ensemble we essentially confirm our findings for the $\mathcal{S}^{2}$ model qualitatively.

In general, $q$ turns out to depend on $T$ such that it increases with $T$ towards the isotropic-cholesteric phase transition. At higher $\varepsilon_{1}$ our data for $q$ agree increasingly well with the theoretical prediction $q_{0}$ which we develop in this work. The expression we derive includes the formula proposed by Luckhurst et al. [15] as a special case which reduces further to the one obtained by van der Meer et al. [14].

However, regardless of whether one uses a lattice or an off-lattice model, the implementation of boundary conditions is particularly crucial for the simulation of chiral systems. This is because the formation of a cholesteric helix introduces a new length scale (namely the magnitude of $p$ ) besides the dimensions of the computational cell. Any mismatch between these two length scales would put the cholesteric helix under an artificial stress. Therefore, specialised boundary conditions are required to avoid these spurious effects.

In this work we employ the so-called "spiralling algorithm" devised by Saslow and coworkers [35, 36] for spin-lattice models. This algorithm, which is also suitable for the $\mathcal{S}^{1}$ - and $\mathcal{S}^{2}$-model investigated here, is utilised in the $(z$-)direction in which the cholesteric helix evolves; in the other two directions the conventional toroidal boundary conditions are utilised. For the off-lattice model we propose here a variant of this algorithm which is needed to implement the minimum image convention for shortrange interactions [33, 37].

An important criterion for the correctness of the implementation of the spiralling algorithm is the translational invariance of the local nematic order parameter. Unfortunately, no such demonstration is provided by either Luckhurst et al. [15] nor by Memmer and Janssen [16]. The latter authors prefer to use, at least in our view, un- 
necessarily complicated boundary conditions.

We have also tried to implement the boundary conditions as described by Luckhurst et al. [15] which somehow requires a permanent recalculation of $q$ just for the boundary conditions. This procedure is not an integral part of Saslow's original spiralling algorithm [35, 36]. In fact, our implementation of the algorithm proposed by Luckhurst et al. [15] yields a local nematic order parameter which is not translationally invariant but is lower towards the ends of the computational cell compared with a nearly constant value around its mid section. From this observation we conclude that (at least in our implementation) the algorithm used in the simulations of Luckhurst et al. does not work properly.

\section{Acknowledgement(s)}

This article is dedicated to the memory of the late Professor Gerhard H. Findenegg, outstanding scientist and inspiring academic teacher. One of us (M. S.) mourns the loss of a wonderful colleague, and, above all, of a dear friend. It was a privilege and honour to know Gerhard personally.

\section{Appendix A. Expansions in the basis of rotational invariants}

The purpose of this appendix is to present details of the expansion of the interaction potential for our general model introduced in Section 2.1. As indicated in Section 2.1 the expansion of $u$ can be carried out most conveniently in the basis of rotational invariants $\left\{\mathcal{S}_{l_{1} l_{2} l}^{n_{1} n_{2}}\right\}$. These basis functions are defined as [19]

$$
\begin{aligned}
\mathcal{S}_{l_{1} l_{2} l}^{n_{1} n_{2}}\left(\Omega_{1}, \Omega_{2}, \Omega\right)= & i^{l_{1}-l_{2}-l} \sum_{m_{1} m_{2} m}\left(\begin{array}{ccc}
l_{1} & l_{2} & l \\
m_{1} & m_{2} & m
\end{array}\right) \\
& \times \mathcal{D}_{m_{1} n_{1}}^{l_{1} *}\left(\Omega_{1}\right) \mathcal{D}_{m_{2} n_{2}}^{l_{2} *}\left(\Omega_{2}\right) \mathcal{D}_{m 0}^{l^{*}}(\Omega),
\end{aligned}
$$

where $i \equiv \sqrt{-1}$ is the imaginary number, the asterisk indicates the complex conjugate, the term in parentheses is a Wigner $3 j$ symbol, and

$$
\mathcal{D}_{m n}^{l}(\Omega)=\exp (-i m \phi) d_{m n}^{l}(\theta) \exp (-i n \chi)
$$

is a Wigner rotation matrix $[18,42]$. In Equations (A1) and (A2), $l^{\prime}$ (that is, $l_{1}, l_{2}$, or $l$ ) is a non-negative integer and the integers $m^{\prime}$ and $n^{\prime}$ are related to $l^{\prime}$ such that $m^{\prime}, n^{\prime} \in\left[-l^{\prime}, l^{\prime}\right]$. Thus, for each $l^{\prime}$ one needs to consider $2 l^{\prime}+1$ integers $m^{\prime}$ and $n^{\prime}$. Therefore, as it stands, the expansion in Equation (3) involves an overwhelming number of terms even for small values of $l_{1}, l_{2}$, and $l$. This makes Equation (3) virtually useless from a practical perspective (albeit it is formally exact) without introducing additional assumptions.

Because of the cylindrical symmetry of $\widehat{\boldsymbol{r}}_{12}$, the index $n$ of the third Wigner rotation matrix on the right-hand side of Equation (A1) has been put to zero. This allows us to replace $\mathcal{D}_{m 0}^{l^{*}}$ by the spherical harmonic $\mathcal{Y}_{l m}$ through Equation (A.105) of the book by Gray and Gubbins [18]. Taking the $z$-axis of the reference frame to coincide with $\widehat{\boldsymbol{r}}_{12}$ we can also employ Equation (A.55) of that same reference which immediately suggests 
that $l=m=0$ in Equation (A1). This latter equation can then be rewritten as

$$
\mathcal{S}_{l l 0}^{n_{1} n_{2}}\left(\Omega_{1}, \Omega_{2}, \Omega\right)=\sum_{m}\left(\begin{array}{ccc}
l & l & 0 \\
m & \underline{m} & 0
\end{array}\right) \mathcal{D}_{m n_{1}}^{l *}\left(\Omega_{1}\right) \mathcal{D}_{\underline{m} n_{2}}^{l *}\left(\Omega_{2}\right),
$$

where we keep the argument $\Omega$ only formally, have used the fact that $\mathcal{D}_{00}^{0}=1$, and exploited the selection rules

- $\left|l_{1}-l_{2}\right| \leq l \leq l_{1}+l_{2}$ (triangle inequality)

- $m_{1}+m_{2}=m$

for non-zero Wigner $3 j$ symbols. In addition, we introduced the shorthand notation $m \equiv-m$.

We now restrict the subsequent discussion to linear mesogens which implies $n_{1}=$ $n_{2}=0$. For this special case the Euler angle $\chi^{\prime}$ is redundant such that $\chi^{\prime}$ can be put to zero without loss of generality. Consider now an inversion of mesogen 1 or 2 such that $\theta^{\prime} \rightarrow \pi-\theta^{\prime}$ and $\phi^{\prime} \rightarrow \phi^{\prime}+\pi$. From the definition of the Wigner rotation matrices [see Equation (A2)] it is easy to verify that

$$
\mathcal{D}_{m^{\prime} 0}^{l^{\prime}}\left(\pi-\theta^{\prime}, \phi^{\prime}+\pi, 0\right)=(-)^{l^{\prime}} \mathcal{D}_{m^{\prime} 0}^{l^{\prime}}\left(\theta^{\prime} \phi^{\prime} 0\right)
$$

where we introduced the shorthand notation $(-) \equiv-1$ To arrive at this expression we used the fact that $\exp \left[-i m\left(\phi^{\prime}+\pi\right)\right]=(-)^{m} \exp \left(-i m \phi^{\prime}\right)$ and the relation (see Equation (A.67) of Ref. [18])

$$
d_{m^{\prime} 0}^{l^{\prime}}\left(\pi-\theta^{\prime}\right)=(-)^{l^{\prime}} d_{\underline{m}^{\prime} 0}^{l^{\prime}}\left(\theta^{\prime}\right)=(-)^{l^{\prime}+m^{\prime}} d_{m^{\prime} 0}^{l^{\prime}}\left(\theta^{\prime}\right) .
$$

A comment applies at this point. The assumed spheroidal symmetry of our mesogens cannot be realised in any chiral chemical substance synthesised in the laboratory. Because in our chiral model system, in which only the symmetry between $\boldsymbol{r}_{12}$ and $-\boldsymbol{r}_{12}$ is broken by hand as it were (see Section 2.1.2), the assumption of spheroidal mesogens does not constitute a contradiction.

Because of Equation (A3), Equation (A4) suggests that $l_{1}$ and $l_{2}$ must be even (including the even integer 0). We limit Equation (3) to $0 \leq l^{\prime} \leq 2$ to minimise the number of terms in the expansion in Equation (3) as much as possible.

We can therefore rewrite Equation $(\mathrm{A} 1)$ as $\left(\mathcal{D}_{00}^{0}=1\right)$

$$
\begin{aligned}
\mathcal{S}_{l l 0}^{00}\left(\Omega_{1}, \Omega_{2}, \Omega\right) & =\frac{1}{\sqrt{2 l+1}} \sum_{m}(-)^{m} \mathcal{D}_{m 0}^{l^{*}}\left(\Omega_{1}\right) \mathcal{D}_{\underline{m} 0}^{l^{*}}\left(\Omega_{2}\right) \\
& =\frac{1}{\sqrt{2 l+1}}\left(\frac{4 \pi}{2 l+1}\right) \sum_{m}(-)^{m} \mathcal{Y}_{l m}\left(\omega_{1}\right) \mathcal{Y}_{l \underline{m}}\left(\omega_{2}\right) \\
& =\frac{1}{\sqrt{2 l+1}}\left(\frac{4 \pi}{2 l+1}\right) \sum_{m} \mathcal{Y}_{l m}\left(\omega_{1}\right) \mathcal{Y}_{l m}^{*}\left(\omega_{2}\right) \\
& =\frac{1}{\sqrt{2 l+1}} P_{l}(y),
\end{aligned}
$$

where $P_{l}$ is the $l$-th Legendre polynomial and $y \equiv \cos \gamma_{12}=\widehat{\boldsymbol{u}}_{1} \cdot \widehat{\boldsymbol{u}}_{2}$. To arrive at the final result in Equation (8), we employed Equations (A.33) and (A.105) of the book by Gray and Gubbins [18] as well as the Condon-Shortley phase convention [43]. 
To derive an expression for $\mathcal{S}_{221}$ suitable for computer simulations we proceed as follows. Suppose we are given three vectors $\boldsymbol{a}, \boldsymbol{b}$, and $\boldsymbol{c}$. The sign of the scalar triple product $\boldsymbol{a} \cdot(\boldsymbol{b} \times \boldsymbol{c})$ changes depending on whether the three vectors form a left- or righthanded system whereas $\|\boldsymbol{a} \cdot(\boldsymbol{b} \times \boldsymbol{c})\|$ remains unaltered. The scalar triple product also remains unaltered upon cyclic permutation of the three vectors. The mathematical character of the scalar triple product is exploited to break the symmetry such that the interaction potential loses its invariance with respect to the transformation $\widehat{\boldsymbol{r}}_{12} \rightarrow-\widehat{\boldsymbol{r}}_{12}$.

According to Stone [19] we can express the scalar triple product via

$$
\mathcal{S}_{111}\left(\Omega_{1}, \Omega_{2}, \Omega\right)=\sqrt{\frac{1}{6}}\left(\widehat{\boldsymbol{u}}_{1} \times \widehat{\boldsymbol{u}}_{2}\right) \cdot \widehat{\boldsymbol{r}}_{12} .
$$

Consider now the expression

$$
\mathcal{S}_{110} \mathcal{S}_{111}=-\frac{1}{3} \frac{1}{\sqrt{2}}\left(\widehat{\boldsymbol{u}}_{1} \cdot \widehat{\boldsymbol{u}}_{2}\right)\left(\widehat{\boldsymbol{u}}_{1} \times \widehat{\boldsymbol{u}}_{2}\right) \cdot \widehat{\boldsymbol{r}}_{12},
$$

where Table 2 of the paper by Stone [19] has also been used. For the time being, we dropped the arguments of the rotational invariants to ease the notational burden. Clearly, this quantity changes as if $\widehat{\boldsymbol{r}}_{12} \rightarrow-\widehat{\boldsymbol{r}}_{12}$ but preserves the invariance of the interaction potential if $\widehat{\boldsymbol{u}}_{i} \rightarrow-\widehat{\boldsymbol{u}}_{i}$ (with $i$ being either 1 or 2 ). If the orientation dependence of the interaction between a pair of mesogens is described by Equation (A7), the formation of a cholesteric helix is enabled while at the same time the nematic order is preserved in planes perpendicular to the propagation direction of the helix.

To evaluate Equation (A7) we take recourse to the product formula for rotational invariants [19]. Applying it we obtain

$$
\begin{aligned}
\mathcal{S}_{110} \mathcal{S}_{111}= & \sum_{l_{1} l_{2} l} i^{1-1-0+1-1-1+l_{1}-l_{2}-l}\left(2 l_{1}+1\right)\left(2 l_{2}+1\right)(2 l+1) \\
& \times\left(\begin{array}{ccc}
1 & 1 & l_{1} \\
0 & 0 & 0
\end{array}\right)\left(\begin{array}{ccc}
1 & 1 & l_{2} \\
0 & 0 & 0
\end{array}\right)\left(\begin{array}{ccc}
0 & 1 & l \\
0 & 0 & 0
\end{array}\right) \\
& \times\left\{\begin{array}{ccc}
1 & 1 & l_{1} \\
1 & 1 & l_{2} \\
0 & 1 & l
\end{array}\right\} \mathcal{S}_{l_{1} l_{2} l}
\end{aligned}
$$

where the quantity in curly brackets is a Wigner $9 j$ symbol. From the parity selection rule for the Wigner $3 j$ symbols (see Equations (A.139) and (A.155) of Ref. [18]) we conclude that $l_{1}$ and $l_{2}$ must be even and that they can assume the values 0 and 2 independently whereas $l=1$ is the only possibility.

To evaluate the Wigner $9 j$ symbol we realise that it assumes a non-zero value if and only if each of its three rows and columns satisfy the triangle inequality. Applying this logic to the last column of the Wigner $9 j$ symbol immediately suggests that $l_{1}=l_{2}=2$ is the only option (because $l_{1}$ and $l_{2}$ must not be odd). Using $\mathcal{S}_{110}$ from Table 2 and Equation (2.16) of the paper by Stone [19] it is straightforward to verify that

$$
\mathcal{S}_{221}\left(\Omega_{1}, \Omega_{2}, \Omega\right)=-\sqrt{\frac{3}{10}}\left(\widehat{\boldsymbol{u}}_{1} \cdot \widehat{\boldsymbol{u}}_{2}\right)\left(\widehat{\boldsymbol{u}}_{1} \times \widehat{\boldsymbol{u}}_{2}\right) \cdot \widehat{\boldsymbol{r}}_{12} .
$$




\section{References}

[1] B.G.g. Chen, P.J. Ackerman, G.P. Alexander, R.D. Kamien and I.I. Smalyukh, Phys. Rev. Lett. 110, 237801 (2013).

[2] P.J. Ackerman, R.P. Trivedi, B. Senyuk, J. van de Lagemaat and I.I. Smalyukh, Phys. Rev. E 90 (1), 012505 (2014).

[3] H.K. Bisoyi and Q. Li, Acc. Chem. Res. 47 (10), 3184-3195 (2014).

[4] A. Nych, J.i. Fukuda, U. Ognysta, S. Žumer and I. Muševič, Nat. Phys. 13 (12), 1215-1220 (2017).

[5] I. Dierking, Symmetry 6 (2), 444-472 (2014).

[6] J. Kobashi, H. Yoshida and M. Ozaki, Nat. Photon. 10 (6), 389-392 (2016).

[7] H. Coles and S. Morris, Nat. Photon. 4 (10), 676-685 (2010).

[8] N.Y. Ha, Y. Ohtsuka, S.M. Jeong, S. Nishimura, G. Suzaki, Y. Takanishi, K. Ishikawa and H. Takezoe, Nat. Mat. 7 (1), 43-47 (2008).

[9] B.P. Huff, J.J. Krich and P.J. Collings, Phys. Rev. E 61, 5372-5378 (2000).

[10] R.S. Pindak, C.C. Huang and J.T. Ho, Phys. Rev. Lett. 32, 43-46 (1974).

[11] S. Varga and G. Jackson, Chem. Phys. Lett. 377, 6-12 (2003).

[12] Z. Dogic and S. Fraden, Langmuir 16, 7820-7824 (2000).

[13] H.H. Wensink and G. Jackson, J. Chem. Phys. 130 (23), 234911 (2009).

[14] B.W. van der Meer, G. Vertogen, A.J. Dekker and J.G.J. Ypma, J. Chem. Phys. 65, 3935-3943 (1976).

[15] G.R. Luckhurst, S. Romano and H.B. Zewdie, J. Chem. Soc. Faraday Trans. 92, 17811791 (1996).

[16] R. Memmer and F. Janssen, J. Chem. Soc. Faraday Trans. 94, 267-276 (1998).

[17] R. Memmer and F. Janssen, Liq. Cryst. 24, 805-809 (1998).

[18] C.G. Gray and K.E. Gubbins, Theory of Molecular Fluids, Vol. 1 (Clarendon Press, Oxford, 1984).

[19] A.J. Stone, Mol. Phys. 36, 241-256 (1978).

[20] W. Maier and A. Saupe, Z. Naturforsch. A 15, 287-292 (1960).

[21] R. Memmer, H.G. Kuball and A. Schönhofer, Liq. Cryst. 15, 345-360 (1993).

[22] M. Melle, M. Theile, C.K. Hall and M. Schoen, Int. J. Mol. Sci. 14, 17584-17607 (2013).

[23] S. Varga and G. Jackson, Mol. Phys. 104, 3681-3691 (2006).

[24] L. Blum and A.J. Torruella, J. Chem. Phys. 56, 303-310 (1972).

[25] A.J. Stone, Mol. Phys. 33, 293-299 (1977).

[26] L.D. Barron and A.D. Buckingham, J. Am. Chem. Soc. 96, 4769-4773 (1974).

[27] W.J.A. Goossens, Mol. Cryst. Liq. Cryst. 12, 237-244 (1971).

[28] R. Eppenga and D. Frenkel, Mol. Phys. 52, 1303-1334 (1984).

[29] P. Oswald and P. Pieranski, Nematic and cholesteric liquid crystals: Concepts and physical properties illustrated by experiments (CRC Press, Boca Raton, 2005).

[30] R.A. Skutnik, L. Lehmann, S. Püschel-Schlotthauer, G. Jackson and M. Schoen, Mol. Phys. 117, 2830-2845 (2019).

[31] D. Frenkel and R. Eppenga, Phys. Rev. A 31, 1776-1787 (1985).

[32] J.-C. Eichler, R.A. Skutnik, A. Sengupta, M.G. Mazza and M. Schoen, Mol. Phys. 117, 3715-3733 (2019).

[33] M.P. Allen and D.J. Tildesley, Computer simulation of liquids, 2nd ed. (Oxford University Press, Oxford, 2017).

[34] M.P. Allen and A.J. Masters, Mol. Phys. 79, 277-289 (1993).

[35] W.M. Saslow, M. Gabay and W.M. Zhang, Phy. Rev. Lett. 68, 3627-3630 (1992).

[36] M. Collins and W.M. Saslow, Phys. Rev. B 53, 8533-8538 (1996).

[37] D. Frenkel and B. Smit, Understanding molecular simulation: from algorithms to applications (Academic Press, San Diego, 2001).

[38] C. Chiccoli, P. Pasini and C. Zannoni, Liq. Cryst. 2, 39-54 (1987).

[39] D.A. McQuarrie, Statistical mechanics (Harper \& Row, New York, 1976).

[40] M. Greschek and M. Schoen, Phys. Rev. E 83, 011704 (2011). 
[41] Y.R. Lin-Liu and M.A. Lee, Phys. Rev. A 28, 2580-2583 (1983).

[42] D.M. Brink and G.R. Satchler, Angular momentum, 2nd ed. (Clarendon Press, Oxford, 1968).

[43] G.B. Arfken and H.J. Weber, Mathematical methods for physicists, 6th ed. (Elsevier, San Diego, USA, 2005), p. 270. 